\title{
An Observational Determination of the Bolometric Quasar Luminosity Function
}

\section{Citation}

Hopkins, Philip F., Gordon T. Richards, and Lars Hernquist. 2007. "An Observational Determination of the Bolometric Quasar Luminosity Function." The Astrophysical Journal 654 (2): 731-53. https://doi.org/10.1086/509629.

\section{Permanent link}

http://nrs.harvard.edu/urn-3:HUL.InstRepos:41381596

\section{Terms of Use}

This article was downloaded from Harvard University's DASH repository, and is made available under the terms and conditions applicable to Other Posted Material, as set forth at http:// nrs.harvard.edu/urn-3:HUL.InstRepos:dash.current.terms-of-use\#LAA

\section{Share Your Story}

The Harvard community has made this article openly available.

Please share how this access benefits you. Submit a story.

Accessibility 
Submitted to ApJ, May 23, 2006

Preprint typeset using $\mathrm{LT}_{\mathrm{E}} \mathrm{X}$ style emulateapj v. 6/22/04

\title{
AN OBSERVATIONAL DETERMINATION OF THE BOLOMETRIC QUASAR LUMINOSITY FUNCTION
}

\author{
PHILIP F. HopKINS ${ }^{1}$, GORdON T. RICHARds ${ }^{2}, \&$ LARS HERNQUIST $^{1}$ \\ Submitted to ApJ, May 23, 2006
}

\begin{abstract}
We combine a large set of quasar luminosity function (QLF) measurements from the rest-frame optical, soft and hard X-ray, and near- and mid-infrared bands to determine the bolometric QLF in the redshift interval $z=0-6$. Accounting for the observed distributions of quasar column densities and variation of spectral energy distribution (SED) shapes, and their dependence on luminosity, makes it possible to integrate the observations in a reliable manner and provides a baseline in redshift and luminosity larger than that of any individual survey. We infer the QLF break luminosity and faint-end slope out to $z \sim 4.5$ and confirm at high significance $(\gtrsim 10 \sigma)$ previous claims of a flattening in both the faint- and bright-end slopes with redshift. With the best-fit estimates of the column density distribution and quasar SED, which both depend on luminosity, a single bolometric QLF self-consistently reproduces the observed QLFs in all bands and at all redshifts for which we compile measurements. Ignoring this luminosity dependence does not yield a self-consistent bolometric QLF and there is no evidence for any additional dependence on redshift. We calculate the expected relic black hole mass function and mass density, cosmic X-ray background, and ionization rate as a function of redshift and find they are consistent with existing measurements. The peak in the total quasar luminosity density is well-constrained at $z=2.15 \pm 0.05$. We provide a number of fitting functions to the bolometric QLF and its manifestations in various bands, and a script ${ }^{3}$ to return the QLF at arbitrary frequency and redshift from these fits.
\end{abstract}

Subject headings: quasars: general — galaxies: active — galaxies: evolution — galaxies: luminosity function - cosmology: observations - X-rays: galaxies — infrared: galaxies — ultraviolet: galaxies

\section{INTRODUCTION}

Determining the nature and cosmological evolution of the quasar luminosity function (QLF) has been of interest since quasars were first identified as cosmological sources (Schmidt 1968), and understanding the QLF is crucial to inferring the formation history of supermassive black holes, as well as the buildup of cosmic X-ray and infrared (IR) backgrounds and the contribution of quasars to reionization. Furthermore, the recognition that black holes appear to reside at the centers of most galaxies (e.g., Kormendy \& Richstone 1995) and that the masses of these black holes are correlated with either the mass (Magorrian et al. 1998) or the velocity dispersion (Ferrarese \& Merritt 2000; Gebhardt et al. 2000) of their host spheroids, demonstrates a link between the origin of galaxies and supermassive black holes. Hence, determining the evolution of the QLF is also critical to understanding galaxy formation and evolution.

The study of the QLF has a long history (e.g., Schmidt \& Green 1983; Koo \& Kron 1988; Boyle et al. 1988; Hewett et al. 1993; Hartwick \& Schade 1990; Warren et al. 1994; Schmidt et al. 1995; Kennefick et al. 1995; Pei 1995), but in recent years, surveys such as the Two Degree Field (2dF) QSO Redshift Survey (2QZ; Bovle et al. 2000) and the Sloan Digital Sky Survey (SDSS; York et al. 2000) have provided large, homogeneous quasar samples over the range of redshifts $z=0-6$ (e.g., Bovle et al. 2000; Fan et al. 2001a, 2004; Croom et al. 2004; Richards et al. 2005, 2006b; Jiang et al. 2006a). In addition, a great deal of information on the X-ray and infrared properties of quasars has become

\footnotetext{
${ }^{1}$ Harvard-Smithsonian Center for Astrophysics, 60 Garden Street, Cambridge, MA 02138

2 Department of Physics and Astronomy, The Johns Hopkins University, 3400 North Charles Street, Baltimore, MD 21218

3 http://www.cfa.harvard.edu/ phopkins/Site/qlf.html
}

available, and surveys with e.g. Chandra, XMM, ROSAT, and Spitzer have enabled studies of the evolution of the QLF across many frequencies (e.g., Mivaii et al. 2000; Ueda et al. 2003; Haas et al. 2004; Barger et al. 2005; Hasinger et al. 2005; Brown et al. 2006; Matute et al. 2006).

Surprising and suggestive trends have emerged from these studies. For example, both the spectral shapes (e.g., Wilkes et al. 1994; Green et al. 1995; Vignali et al. 2003; Strateva et al. 2005; Richards et al. 2006c; Steffen et al. 2006) and column density distributions (e.g., Hill, Goodrich, \& DePov 1996; Simpson. Rawlings, \& Lacv 1999; Willott et al. 2000; Simpson \& Rawlings 2000; Steffen et al. 2003; Ueda et al. 2003; Grimes, Rawlings, \& Willott 2004; Hasinger 2004; Sazonov \& Revnivtsev 2004; Barger et al. 2005; Simpson 2005; Hao et al. 2005) of quasars appear to depend systematically on luminosity, with the brightest quasars being the least obscured and the most dominated by the optical/UV portion of the spectrum. X-ray studies (e.g., Page et al. 1997; Mivaii et al. 2000, 2001; La Franca et al. 2002; Cowie et al. 2003; Ueda et al. 2003; Fiore et al. 2003; Barger et al. 2003b; Hasinger et al. 2005) and some optical, radio, and IR measurements (e.g., Hunt et al. 2004; Cirasuolo et al. 2005; Matute et al. 2006) indicate that the space density of low luminosity active galactic nuclei (AGN) peaks at redshifts lower than that of bright quasars, specifically observing a flattening of the QLF faint-end slope with redshift. It has been argued that this follows a similar pattern of "cosmic downsizing" as has recently been observed in galaxy spheroid populations (e.g., Cowie et al. 1996). Similarly, the brightend slope of the QLF appears to become shallower towards higher redshifts, from both direct measurements (Fan et al. 2001b, 2003; Richards et al. 2006b) and (albeit weaker) constraints from gravitational lensing (Comerford et al. 2002; Wvithe \& Loeb 2002; Wvithe 2004; Richards et al. 2006a). 
Various models have been proposed to explain these trends, many of which postulate that feedback from black hole growth plays a key role in determining the black holehost galaxy (e.g. $M_{\mathrm{BH}}-\sigma$ ) relationships (Silk \& Rees 1998; Di Matteo et al. 2005), and co-evolution of black holes and their host spheroids. The evolution of the faint and brightend slopes may be linked to these processes, with AGN feedback providing the mechanism for cosmic downsizing, shutting down the growth of the most massive systems at high redshift (e.g., Merloni 2004; Granato et al. 2004; Hopkins et al. 2006b; Croton et al. 2006) and potentially steepening the lowredshift bright-end QLF slope as a result (Scannapieco \& Oh 2004), while this feedback-driven quasar decay determines the shape of the faint-end QLF (Hopkins et al. 2006a). Feedback may also explain trends in obscuration with luminosity, either through dust sublimation (e.g., Lawrence 1991) or expulsion of gas and dust on galactic scales (e.g., Hopkins et al. 2005d). But attempts to quantitatively link the downsizing of quasar and galaxy populations, both theoretically (Hopkins et al. 2006b) and observationally (e.g., Hopkins et al. 2006d), depend on a reliable determination of the QLF.

However, inferences drawn from the observed trends suffer from complications arising from various biases. For example, optical quasar surveys generally probe large volumes $\left(\sim 10,000 \mathrm{deg}^{2}\right)$, enabling uniform sample selection at many redshifts and the discovery of the brightest quasars, but miss substantial populations of obscured (e.g., Risaliti et al. 1999; Ueda et al. 2003; Treister et al. 2004) or heavily reddened (e.g., Webster et al. 1995; Richards et al. 2001; Brotherton et al. 2001; Gregg et al. 2002; Hopkins et al. 2004) quasars, and generally cover a relatively small baseline $(\sim 1-2$ dex) in luminosity. Several seminal efforts (upon which we seek to expand) have been made to extend these baselines by compiling various optical quasar observations (e.g., Hartwick \& Schade 1990; Warren et al. 1994), most notably Pei (1995), but these have still been severely limited by obscuration/reddening and span only $\sim 2-2.5$ dex in luminosity, and furthermore the area and depth of quasar surveys have since increased by orders of magnitude. Hard X-ray and IR samples provide a more complete census of obscured quasars, and sample much fainter luminosities and wider ( $\sim 4-5 \mathrm{dex})$ luminosity ranges, but are measured from much smaller survey areas $\left(\lesssim 1 \mathrm{deg}^{2}\right)$. Without carefully accounting for the differential effects of obscuration, different spectral shapes, and selection effects across bands, it is not clear if trends observed primarily at a single frequency are physically meaningful or robust across frequencies. Furthermore, theoretical models typically do not deal directly with the quasar luminosity in a given band, but instead treat the bolometric quasar luminosity, and it is not clear that a simple bolometric correction can reliably translate between the two - the effects above may change the observed QLF shape as a function of frequency, luminosity, and redshift.

In this paper, we combine recent measurements of the QLF in many wavelengths from the mid-IR through hard $\mathrm{X}$-rays, to determine the observed bolometric quasar luminosity function. By utilizing multiwavelength measurements of quasar SEDs (Elvis et al. 1994; Vanden Berk et al. 2001; Telfer et al. 2002; Vignali et al. 2003; Fan et al. 2003; Hatziminaoglou et al. 2005; Richards et al. 2006c; Shemmer et al. 2006) that probe the distribution of obscuration up to and including Compton-thick column densities (Risaliti et al. 1999; Ueda et al. 2003; Treister et al. 2004;
Mainieri et al. 2005; Hao et al. 2005; Tozzi et al. 2006), we can predict the QLFs that would be observed as a function of luminosity, frequency, and redshift from a given bolometric QLF and compare these simultaneously with all observed QLFs. This makes it possible to constrain the shape and evolution of the QLF over $\sim 8$ orders of magnitude in luminosity and $\sim 9$ in space density, from $z=0-6$, larger than the baselines probed by any individual survey.

In $\S 2$ we describe the observational data sets, including the observed column density distributions ( $\$ 2.2$ ) and SEDs adopted $(\$ 2.1)$, and consider in detail the consistency of the QLF across various frequencies given different simplifications of these distributions $(\S 2.3$. In $\S 3$ we calculate the bolometric QLF as a function of luminosity and redshift, and consider the detailed evolution of the QLF shape and its manifestation in different observed bands. In $\S 4$ we consider a number of complementary constraints, testing models of quasar lifetimes, the evolution in the QLF shape, and the buildup of the black hole population ( $\$ 4.1$, and we calculate the relic black hole mass function, cosmic X-ray background $(\$$ 4.2), and ionization rates $(\$ 4.3)$ expected from the bolometric QLF and compare these to observations. In $\S 5$ we summarize and discuss our results and future prospects for improving the measurements.

We adopt a $\Omega_{\mathrm{M}}=0.3, \Omega_{\Lambda}=0.7, H_{0}=70 \mathrm{~km} \mathrm{~s}^{-1} \mathrm{Mpc}^{-1}$ cosmology, consistent with Spergel et al. (2003, 2006). Quasar $B$-magnitudes are Vega, and $L_{\odot}$ refers to the bolometric solar luminosity $L_{\odot} \equiv 3.9 \times 10^{33} \mathrm{erg} \mathrm{s}^{-1}$.

\section{THE OBSERVATIONAL DATA SET}

In what follows, we compile a large number of binned QLF measurements in different redshifts ranges, observed wavebands, and luminosity intervals. Table 1 lists the samples used, with the survey and fields for each, the rest wavelength or band measured, the redshift and luminosity range of the observed quasars, and the number of quasars in the sample. We use the term "quasar" rather loosely throughout, as the traditional $M_{B}<-23$ cut is not readily applicable to multiwavelength observations or obscured sources. Our compilation instead attempts to represent all AGN with intrinsic (obscuration-corrected) luminosities above the observational limits at each redshift. This generally extends to the typically adopted $\sim 10^{42} \mathrm{erg} \mathrm{s}^{-1}$ X-ray luminosity limit $\left(L_{\text {bol }} \sim\right.$ $\left.10^{10} L_{\odot}\right)$, below which confusion with normal star-forming and starburst galaxies becomes problematic (although resolved hosts at low redshift extend this to $\sim 10^{9} L_{\odot}$; e.g., Hao et al. 2005).

Some of the observational estimates are derived from the same sets of observations, or different updates of the same data sets. To avoid "double-counting," at the few points where two binned QLFs overlap in both luminosity and redshift and are derived from the same sample (at that luminosity and redshift), we discard the less well-constrained (usually less recent) point. This is generally rare, and has a negligible effect on our results. It does reduce significantly the samples of Mivaii et al. (2001), for which the Type 1 QLFs are updated in Hasinger et al. (2005), and Croom et al. (2004), which has many (especially faint) luminosity intervals updated in Richards et al. (2005). Some measured QLFs do not appear in this compilation (e.g., Bovle et al. 2000), as updated versions from the same or expanded samples exist (e.g., Croom et al. 2004). We have re-fit our results with these samples (as well as those of e.g. Pei 1995; Jiang et al. 2006b; Sazonov et al. 2006; Shinozaki et al. 2006; Beckmann et al. 
2006b) and find the differences are negligible.

\subsection{The SED and Bolometric Corrections}

We construct a model "intrinsic" (un-reddened) quasar SED to compare observations in different bands. The template spectrum initially follows that derived in Marconi et al. (2004) and consists of a broken power law in the opticalUV with $\alpha_{\mathrm{O}}=-0.44\left(L_{\nu} \propto \nu^{\alpha_{\mathrm{O}}}\right)$ for $1 \mu \mathrm{m}<\lambda<1300 \AA$ (Vanden Berk et al. 2001), and $\alpha_{\mathrm{UV}}=-1.76$ from $1200-$ $500 \AA$ (Telfer et al. 2002), essentially the modal spectral slopes for optically bright blue quasars (Richards et al. 2006c, appropriate given that this is supposed to be a pre-reddened spectrum). Because a number of slightly different bands have been used in optical quasar surveys (e.g. $B, g, i, 1450 \AA$; see Table 10, we further overlay the template spectrum of Richards et al. (2006c) derived from the optically blue (i.e. un-reddened) subsample onto this broken power-law. Technically, we factor out the best-fit power-law in the given range from the Richards et al. (2006c) spectrum and then multiply our template by the residuals, but this makes little difference compared to adopting the Richards et al. (2006c) spectrum over this range. We do the same with the template spectrum of Vanden Berk et al. (2001) for optical wavelengths $1 \mu \mathrm{m}<\lambda<1300 \AA$, if a detailed correction from an observed band to a more "standard" band is needed, but such corrections have generally been provided for the QLF measurements in Table 1

Longwards of $\lambda>1 \mu \mathrm{m}$, we adopt the mean spectrum from Richards et al. (2006c), with a typical observed IR "bump" from reprocessing, eventually truncated as a Rayleigh-Jeans tail of blackbody emission $(\alpha=2)$, and a no-obscuration zero point taken from the optically blue (un-reddened) subsample template spectrum. This gives a $15 \mu \mathrm{m}$ to $R$-band correction similar to the typically adopted $\log \left(L_{15} / L_{R}\right)=0.23$ (e.g., Elvis et al.|1994; Hatziminaoglou et al. 2005; Richards et al. 2006c; Matute et al. 2006; Brown et al. 2006) for optically unobscured (Type 1) quasars.

The X-ray spectrum beyond $0.5 \mathrm{keV}$ is determined by a power-law, with an intrinsic photon index $\Gamma=1.8$ (e.g., George et al. 1998; Perola et al. 2002; Tozzi et al. 2006) and an exponential cutoff at $500 \mathrm{keV}$. A reflection component is included following Ueda et al. (2003), generated with the PEXRAV model (Magdziarz \& Zdziarski 1995) in the XSPEC package with a reflection solid angle of $2 \pi$, inclination $\cos (i)=0.5$ and solar abundances. The $\mathrm{X}$-ray spectrum is then renormalized to a given $\alpha_{\mathrm{ox}} \equiv$ $-0.384 \log \left[L_{\nu}(2500 \AA) / L_{\nu}(2 \mathrm{keV})\right]$, and the points at $500 \AA$ and $50 \AA$ are connected with a power-law. The value of $\alpha_{\text {ox }}$ depends on luminosity (Wilkes et al. 1994; Green et al. 1995; Vignali et al. 2003; Strateva et al.|2005), and we adopt the most recent determination by Steffen et al. (2006),

$$
\alpha_{\text {ox }}=-0.107 \log \left(L_{\nu, 2500} /\left[\mathrm{erg} \mathrm{s}^{-1} \mathrm{~Hz}^{-1}\right]\right)+1.739,
$$

determined specifically for unobscured (Type 1) quasars. The above equation is derived from the least-squares bisector of the $L_{\nu}(2500 \AA)-L_{\nu}(2 \mathrm{keV})$ relation, since neither luminosity can be properly taken as an "independent" variable - the difference if e.g. $L_{\nu}(2500 \AA)$ is considered independent is generally small (see e.g. Steffen et al. 2006), but can be of importance for the most luminous X-ray AGN (yielding a difference of $\sim 30 \%$ in the bolometric correction at $L_{0.5-2 \mathrm{keV}} \sim$ $\left.10^{47} \mathrm{erg} \mathrm{s}^{-1}\right)$. The baseline for these observations is sufficiently large that this relation has been determined for nearly

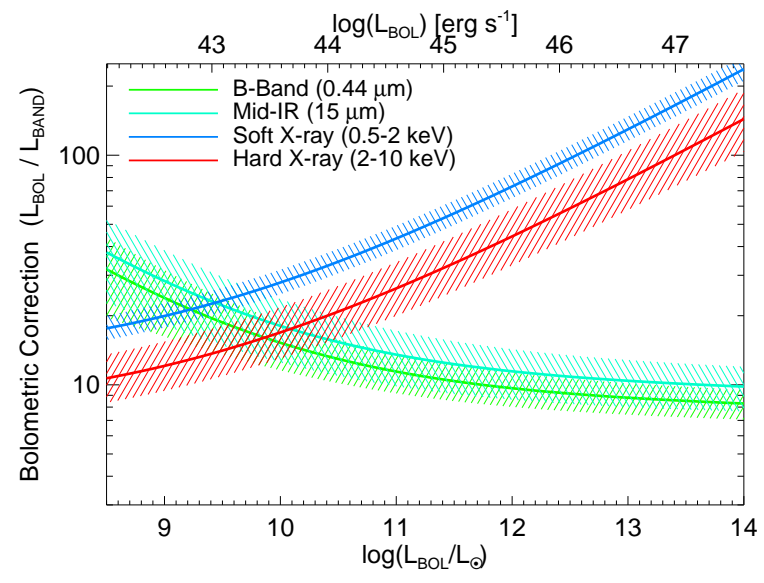

FIG. 1.- Bolometric corrections for $B$-band, mid-IR, soft and hard X-ray bands, determined in $\S 2.1$ from a number of observations as a function of luminosity and given by the fitting formulae in Equation (2). The lognormal dispersion in the distribution of bolometric corrections at fixed $L$, given by Equation 3 is shown as the shaded range for each band. The full quasar template spectrum is available for public download ${ }^{3}$.

all luminosities of interest in the compiled QLF measurements. Recent comparisons between large samples of quasars selected by both optical and X-ray surveys (Risaliti \& Elvis 2005) further suggests that this is an intrinsic correlation, not driven by e.g. the dependence of obscuration on luminosity, as does our comparison of bolometric QLFs derived below. There is no evidence for a trend of $\alpha_{\mathrm{ox}}$ with redshift, or for any other trend in spectral shape with redshift (e.g., Elvis et al. 1994; Vanden Berk et al. 2001; Telfer et al. 2002; Vignali et al. 2003; Fan et al. 2003; Steffen et al. 2006; Richards et al. 2006c; Shemmer et al. 2006, but see also Bechtold et al. 2003), so our spectrum depends only on luminosity.

Ultimately, the bolometric corrections (with zero attenuation) derived can be accurately approximated as a double power-law

$$
\frac{L}{L_{\text {band }}}=c_{1}\left(\frac{L}{10^{10} L_{\odot}}\right)^{k_{1}}+c_{2}\left(\frac{L}{10^{10} L_{\odot}}\right)^{k_{2}},
$$

with $\left(c_{1}, k_{1}, c_{2}, k_{2}\right)$ given by $(6.25,-0.37,9.00,-0.012)$ for $L_{\text {band }}=L_{B}, \quad(7.40,-0.37,10.66,-0.014)$ for $L_{15 \mu \mathrm{m}}, \quad(17.87,0.28,10.03,-0.020)$ for $L_{0.5-2 \mathrm{keV}}$, and $(10.83,0.28,6.08,-0.020)$ for $L_{2}-10 \mathrm{keV}$. The $k_{1}$ term is important when the given portion of the spectrum is not dominant, controlled by the scaling of $\alpha_{\mathrm{ox}}$, and the $k_{2} \approx 0$ term represents the nearly constant bolometric correction when a given portion of the spectrum dominates the bolometric luminosity. Figure 1 shows these corrections as a function of luminosity, which agree broadly with the values in e.g. Richards et al. (2006c) over the luminosity range they consider.

We have generally followed Marconi et al. (2004) in calculating this spectrum, with a more detailed treatment of the optical/IR and a more recent determination of $\alpha_{\mathrm{ox}}$, but this level of detail is ultimately not required. The critical dependence is that of $\alpha_{\mathrm{ox}}$ on luminosity. Adopting the median Richards et al. (2006c) spectrum and rescaling the spectrum upwards of $0.1 \mathrm{keV}$ according to the observed $\alpha_{\text {ox }}$ for different $L_{\nu}(2500 \AA)$ and corresponding bolometric luminosities yields a similar model spectrum and essentially identical conclusions. Likewise, rescaling our median spectrum according 
to Equation (21) in Marconi et al. (2004) gives a similar result. Ignoring the dependence of bolometric corrections on luminosity altogether, however, is problematic. In the optical/UV, it is not as serious, as the bolometric correction changes by only $\sim 20 \%$ from $M_{B}=-23$ to -30 . However, over a $\sim 4$ dex interval in $L_{2-10 \mathrm{keV}}$ comparable to the usual observed baselines, the typical hard and soft X-ray bolometric corrections change by more than an order of magnitude (primarily as given by the $\alpha_{\mathrm{ox}}$-luminosity relation).

Finally, even accounting for the dependence of spectral shape on luminosity, objects with a given luminosity do not all have identical spectra and bolometric corrections. It is important to account for the dispersion in spectral shapes at a given $L$. In general, there will be two components, a correlated and uncorrelated dispersion. If, for example, there is a different spectral slope or value of $\alpha_{\mathrm{ox}}$, then the bolometric correction in certain bands will be larger, but the correction in other bands must be smaller. Also, because the optical/UV contributes a larger fraction of the bolometric luminosity than the X-ray, the resulting dispersion in the $B$-band bolometric correction from different values of $\alpha_{\mathrm{ox}}$ will be smaller than the resulting dispersion in the X-ray bolometric corrections.

We estimate these dispersions in the power-law components of our modeling from the observed distributions, assuming that they are normally distributed; $\sigma_{\alpha_{0}} \approx 0.125$ (Richards et al. 2003, comparing the mean $\alpha_{O}$ in each observed quartile), $\sigma_{\Gamma}=0.30$ (Tozzi et al. 2006), and $\sigma_{\alpha_{0 x}}=$ $0.075-0.14$ (Steffen et al. 2006). Strictly speaking, a larger bolometric correction in one band implies a smaller integrated correction in others, but there are sources of scatter which introduce this effect weakly, such as e.g. variations in line strength in a given band or observational uncertainties in the luminosity in the band. Fitting to the distribution of bolometric corrections in Richards et al. (2006c), after accounting for the luminosity distribution of the sources and correlated dispersions above yields a best-fit uncorrelated dispersion component $\sim 0.1 \mathrm{dex}$, consistent with observational estimates of the scatter in band luminosities owing to these effects (e.g., Elvis et al. 1994; George et al. 1998; Vanden Berk et al. 2001; Perola et al. 2002; Richards et al. 2003; Hopkins et al. 2004). By fitting to a number of Monte Carlo realizations of the spectra as a function of luminosity given these dispersions, we can quantify the effective dispersion in the bolometric correction in different bands as a function of luminosity

$$
\sigma_{\log \left(L / L_{\mathrm{bol}}\right)}=\sigma_{1}\left(L_{\mathrm{bol}} / 10^{9} L_{\odot}\right)^{\beta}+\sigma_{2}
$$

with $\left(\sigma_{1}, \beta, \sigma_{2}\right)=(0.08,-0.25,0.060)$ in the $B$-band, $(0.07,-0.17,0.086)$ in the IR $(15 \mu \mathrm{m}),(0.046,0.10,0.080)$ in the soft X-ray, and $(0.06,0.10,0.08)$ in the hard X-ray. Here, $\sigma_{1}$ and $\beta$ roughly describe the correlated component of the dispersion, $\sigma_{2}$ the uncorrelated component. For typical bright quasars $\left(L_{\text {bol }} \sim 10^{13} L_{\odot}\right)$, this reflects the fact that a significant $(\gtrsim 5 \%$ ) fraction of quasars in any band can have their bolometric luminosities mis-estimated by a factor $\sim 2$ or more by a simple bolometric correction (even one that accounts for the luminosity-dependent spectral shape). These dispersions as a function of luminosity are plotted in Figure 1

\subsection{The Observed Column Density Distribution}

In order to convert an observed luminosity function to a bolometric luminosity function, we must correct for extinction in the different observed bands, which requires the adoption of an observed column density distribution. Essentially, the probability of observing a quasar of a given bolometric luminosity at some observed luminosity in a given band must account for the probability of extinction or attenuation.

We consider three cases. First, our fiducial model adopts the luminosity-dependent observed column density distribution from the hard and soft X-ray observations of Ueda et al. (2003). We also follow Ueda et al. (2003) and include an equal fraction of Compton-thick objects with $N_{\mathrm{H}}>10^{24} \mathrm{~cm}^{-2}$ to that with $N_{\mathrm{H}}=10^{23}-10^{24} \mathrm{~cm}^{-2}$. The evidence for this in Ueda et al. (2003) is tentative, but it produces good agreement with the distribution of Compton-thick column densities subsequently reported by Treister et al. (2004), Mainieri et al. (2005), and Tozzi et al. (2006) and is consistent with upper limits to the obscured fraction from the mid-IR observations of Richards et al. (2006c). Recent very hard X-ray and soft gamma-ray $(\sim 20-200 \mathrm{keV})$ Swift BAT and INTEGRAL observations of local AGN, sensitive even to Compton-thick sources, confirm both a similar Compton-thick fraction and dependence on luminosity (demonstrating also that this trend does not owe to selection effects) (Markwardt et al. 2005; Beckmann et al. 2006a b; Bassani et al. 2006, but see also Wang \& Jiang 2006). Note that this yields a maximum Compton-thick fraction of $\sim 30 \%$ (correcting the observed number density in a sample not sensitive to Compton-thick objects by a factor of 1.4), at low luminosity. This does not imply a uniform factor of 1.4 correction to the quasar space density, as the Compton-thick fraction depends on luminosity in the same manner as the entire column density distribution, and the fraction of Compton-thick sources at high luminosity will in general be much smaller.

Alternatively, we consider a constant (luminosityindependent) column density distribution, again adopting that from Ueda et al. (2003) (fitted to their observations assuming no luminosity dependence). As the opposite extreme, we employ the column density distribution determined in La Franca et al. (2005), which depends on both luminosity and redshift. We discuss these models in $\S 2.3$ but find that they are unable to produce a self-consistent set of luminosity functions in the different observed bands.

Given an $N_{\mathrm{H}}$ distribution, we calculate the extinction at Xray frequencies using the photoelectric absorption cross sections of Morrison \& McCammon (1983) and non-relativistic Compton scattering cross sections. In the optical and midIR, we adopt a canonical gas-to-dust ratio $\left(A_{B} / N_{\mathrm{H}}\right)_{\mathrm{MW}}=$ $8.47 \times 10^{-22} \mathrm{~cm}^{2}$ and Small Magellanic Cloud-like reddening curve from Pei (1992), which observations suggest is appropriate for the majority of reddened quasars (Richards et al. 2003; Hopkins et al. 2004; Ellison et al. 2005), although a Milky-Way like reddening curve changes the optical depth by only $\sim 5-10 \%$ at the wavelengths of interest (excluding the $2100 \AA$ “ “bump”).

Given a bolometric QLF and the observed column density distribution, we can then convolve over the distribution of column densities and spectral shapes at each bolometric luminosity $L$ to infer the implied distribution of luminosities that should be observed in a given band. In other words, knowing the probability of some intrinsic spectral shape and intervening column density, we determine the probability of observing quasars with an intrinsic $L$ at some observed luminosity in the observed band(s).

It is a surprisingly good approximation to this full convolution to adopt an "obscured fraction" as a function of luminosity; i.e. converting the luminosities of the bolometric QLF to luminosities in the observed band using the appro- 
priate bolometric corrections and then multiplying by some "observable fraction" $f(L)$ to correct for the effects of extinction (and the dispersion in spectral shapes). Fitting to this $f(L)$ from our full modeling yields a useful function in comparing optical, soft X-ray, and hard X-ray observations; more directly applicable than the typically calculated fraction with $N_{\mathrm{H}}>10^{22} \mathrm{~cm}^{-2}$. This can be conveniently parameterized as a power law,

$$
f(L) \equiv \frac{\phi\left(L_{i}\right)}{\phi\left(L\left[L_{i}\right]\right)}=f_{46}\left(\frac{L}{10^{46} \mathrm{erg} \mathrm{s}^{-1}}\right)^{\beta},
$$

where $L_{i}$ is the luminosity in some band, and $L$ is the corresponding bolometric luminosity given by the bolometric corrections of Equation (2). This gives values $\left(f_{46}, \beta\right)$ of $(0.260,0.082)$ for $L_{i}=L_{B}(4400 \AA),(0.438,0.068)$ for $L_{i}=$ $L_{I R}(15 \mu \mathrm{m}),(0.609,0.063)$ for $L_{i}=L_{S X}(0.5-2 \mathrm{keV})$, and $(1.243,0.066)$ for $L_{i}=L_{H X}(2-10 \mathrm{keV})$. Note that this "observable fraction" can exceed unity, because the scatter and luminosity dependence of the bolometric corrections significantly changes the shape of the bright-end QLF in the X-ray bands (see Figure 4 below). These simple rescalings are robust for the $B$ and IR bands, with weak dependence on the shape of the bolometric QLF and dispersion in bolometric corrections, but the effects above make the observable fraction in the X-ray bands sensitive to the shape of the QLF; if greater accuracy is required, a more robust fit across a variety of QLF shapes gives $\beta=0.035 \gamma_{2}, 0.034 \gamma_{2}$ for $L_{S X}$ and $L_{H X}$, respectively, for an arbitrary bright-end QLF slope $\gamma_{2}$ (defined in $\$ 3.1$. We caution that these are crude approximations, but the above equations can be used for rough conversion of observed QLFs to bolometric QLFs and vice versa, and for average conversions between bands (multiplying any two such conversions together appropriately).

\subsection{Comparison of the Bolometric QLF Under Different Assumptions}

Given some model for the quasar spectrum and column density distribution, we can calculate the bolometric QLF from observations in some bands. In detail, we convolve a given bolometric QLF over the distribution of intrinsic spectral shapes and column densities, which yields the expected luminosity distribution in some observed band. By integrating, if necessary, over the appropriate redshift and luminosity intervals, we can directly compare this to the binned QLF measurements at each frequency, luminosity, and redshift. We minimize the $\chi^{2}$ of this estimate in relation to the observed QLF in all bands,

$$
\chi^{2} \equiv \sum\left(\frac{\phi_{\text {expected }}\left(L_{\nu}, z \mid \phi_{\text {bol }}\right)-\phi_{\text {obs }}\left(L_{\nu}, z\right)}{\Delta \phi_{\text {obs }}\left(L_{\nu}, z\right)}\right)^{2},
$$

to determine the best-fit bolometric QLF. Before applying this broadly, we would to test our description of the observed spectral shape and column density distributions.

In Figure 2 we show (left panels) the bolometric QLF determined in this manner at several redshifts from our full modeling. At each redshift, we compare with QLF observations from Table 1 with overlapping redshift intervals. We plot the binned QLF measurements rescaled to bolometric points with respect to the best-fit double power law bolometric QLF at that redshift (see $\S 3$ below). In other words, convolving our best fit bolometric QLF with e.g. the quasar spectrum and column density distribution predicts a number density of quasars $n_{\text {mdl }}$ for a given observed bin. Comparing this to the actual number observed, $n_{\mathrm{obs}}$, fixes the ratio $n_{\mathrm{obs}} / n_{\mathrm{mdl}}$. In Figure 2 we show the agreement with all bands simultaneously by plotting $n_{\text {obs }} / n_{\text {mdl }}$ times the best-fit bolometric QLF at each luminosity as the colored points. Given our full SED and obscuration model, the inferred bolometric QLF from each observed waveband agrees well. We quantify this directly, showing in each panel the $\chi^{2} / \nu$ statistic corresponding to the probability that all of the observations from the different bands derive from a single (technically double power-law, although this assumption only weakly changes the $\chi^{2} / \nu$ ) bolometric QLF.

If, however, we simplify our modeling of observed quasar spectra or column densities, the consistency between the QLF implied from the different wavebands is broken. First, we consider the prediction if all quasars had a single spectral shape by adopting the model spectrum of Elvis et al. (1994), with no dependence on luminosity. The optical, IR, and Xray observations are no longer consistent with one another, and the $\chi^{2} / \nu$ at each redshift shown rises by a factor $\sim 4$ to an unacceptably high value. Hard and soft X-ray measurements are still consistent, as they are in both cases related by a relatively straightforward power law. These general conclusions are unchanged regardless of the exact quasar spectrum adopted. In Richards et al. (2006c), mean spectra are computed for the entire quasar sample as well as for several sub-samples: the most optically luminous/dim, optically red/blue, and IR luminous/dim halves (divided at the median values $\log \left[L_{\mathrm{opt}} /\left(\mathrm{erg} \mathrm{s}^{-1}\right)\right]=46.02, \Delta(g-i)=-0.04$, and $\left.\log \left[L_{\mathrm{IR}} /\left(\mathrm{erg} \mathrm{s}^{-1}\right)\right]=46.04\right)$. Figure 3 compares the bolometric QLF as in Figure 2, at $z=1$. For clarity, we show only the best-fit double power-law rather than the binned points. Considering the different Richards et al. (2006c) and Elvis et al. (1994) mean spectra, no single quasar spectrum yields a consistent bolometric QLF at all luminosities (i.e. there is no "effective mean" spectral shape). Taking the mean spectrum of the most optically or IR bright quasars, unsurprisingly, yields a consistent bolometric QLF at the bright end (above the break). The optically and IR dim spectra, on the other hand, are appropriate for lower-luminosity quasars (those near and just below the break), and produce consistent bolometric QLFs in this regime. Note that this does not extend to the lowest luminosities shown, as the "optically dim" objects in Richards et al. (2006c) are still much brighter $\left(L_{\text {bol }} \gtrsim 10^{45} \mathrm{erg} \mathrm{s}^{-1} \approx 3 \times 10^{11} L_{\odot}\right)$ than the lowest luminosities plotted in Figure 3 and probed by $X$-ray samples.

We next examine different assumptions for the column density distribution. In Figure 2 we adopt the full spectral model but consider the luminosity-independent mean column density distribution from Ueda et al. (2003); i.e. a constant ratio of obscured to unobscured AGN $\sim 2: 1$, similar to that found locally (e.g., Risaliti et al. 1999; Hao et al. 2005). Again, the agreement is broken: quasars at low luminosities, where the number of quasars in the Ueda et al. (2003) sample is large, dominate the mean $N_{\mathrm{H}}$ distribution, and as a result this constant obscured fraction provides an acceptable (although still less good) fit to the low-luminosity data. However, at high luminosities, the results derived from different wavebands diverge. The total $\chi^{2} / \nu$ unambiguously rises (factor $\sim 2$ ) at all redshifts, with an increasing discrepancy at higher luminosities (factor $\sim 3$ increase in $\chi^{2} / \nu$ above the QLF break). The disagreement between different bands changes significantly as a function of luminosity, so re-normalizing the constant obscured fraction cannot make the luminosity functions consistent. 


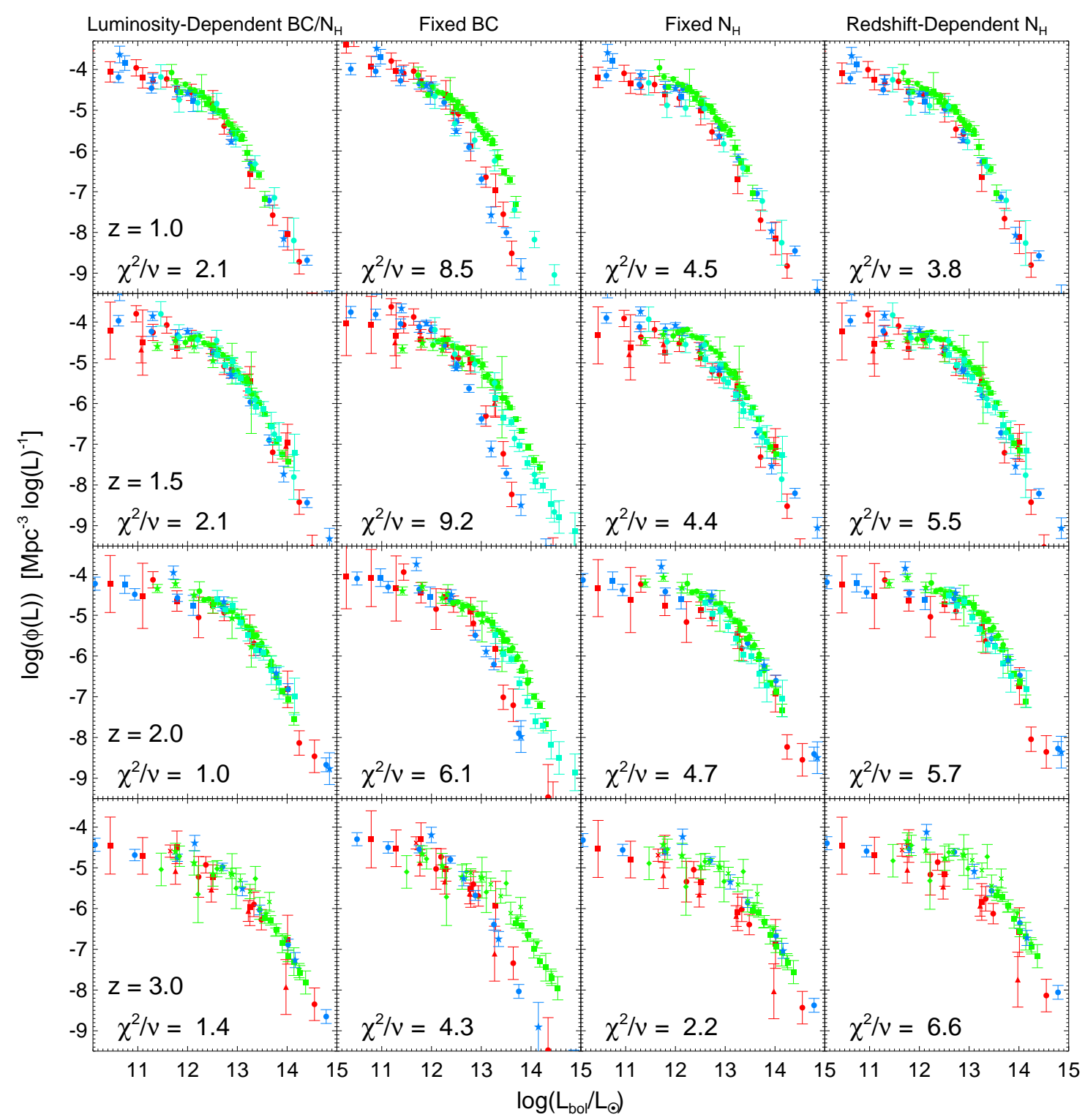

FIG. 2.- The bolometric QLF from the observations in Table 1 at various wavebands (optical: green, soft X-ray: blue, hard X-ray: red, IR: cyan; see Table 11 for the plotting symbols). For ease of comparison, the points in the different bands are rescaled as described in the text to indicate the bolometric QLF implied by each set of measurements. Left panels show the bolometric QLF adopting our full (luminosity-dependent) bolometric corrections and column density distributions (see also Ueda et al. 2003; Marconi et al. 2004; La Franca et al. 2005), at several redshifts (as labeled). Center-left shows the result with the full column distribution, but adopting the constant bolometric corrections of Elvis et al. (1994). Center-right uses the full bolometric corrections, but a luminosity-independent column density distribution (obscured fraction). Right uses the full bolometric corrections and a strongly redshift-dependent column density distribution from La Franca et al. (2005). Each panel shows the reduced $\chi^{2}$ for the assumption that the data yield a consistent bolometric QLF at each redshift. The latter three assumptions do not yield a consistent bolometric QLF at each redshift, unlike for the observationally derived luminosity-dependent bolometric corrections and column density distributions.

Finally, we adopt a column density distribution which evolves strongly with redshift (and luminosity). Specifically, we adopt the fit from La Franca et al. (2005) to the $N_{\mathrm{H}}$ distribution with maximal redshift evolution, similar to the redshift evolution implied by the column density distributions in Tozzi et al. (2006) (fitted assuming maximal redshift dependence and no luminosity dependence), and similar to the redshift evolution of obscured fractions implied by some X-ray background synthesis models (e.g., Comastri et al. 1995; Gilli et al. 1999; but see also Treister \& Urry 2005). The resulting bolometric QLF is shown in the right panels of Figure 2

This fit is reasonable at low and moderate redshifts $z \lesssim 1$ (unsurprisingly, where the observed samples are best con- strained). Extrapolated to higher redshifts, the consistency between different bands breaks down, increasing $\chi^{2} / \nu$ by an unacceptable factor $\sim 3$ by $z \gtrsim 2$. Therefore, while our analysis implies that the obscured fraction must not be constant, it is also clear that any variation is primarily a luminosity, and not a redshift dependence. However, more moderate redshift evolution such as that suggested by Barger et al. (2005) or Ballantyne et al. (2006), with obscured fractions evolving by only $\sim 20 \%$ from $z=0-1$ (with no further evolution at higher redshifts), is consistent with our analysis, and will have only a small impact on the cumulative QLF evolution. Such evolution, being a small effect, would be better constrained by direct measurements of the column density distribution at 


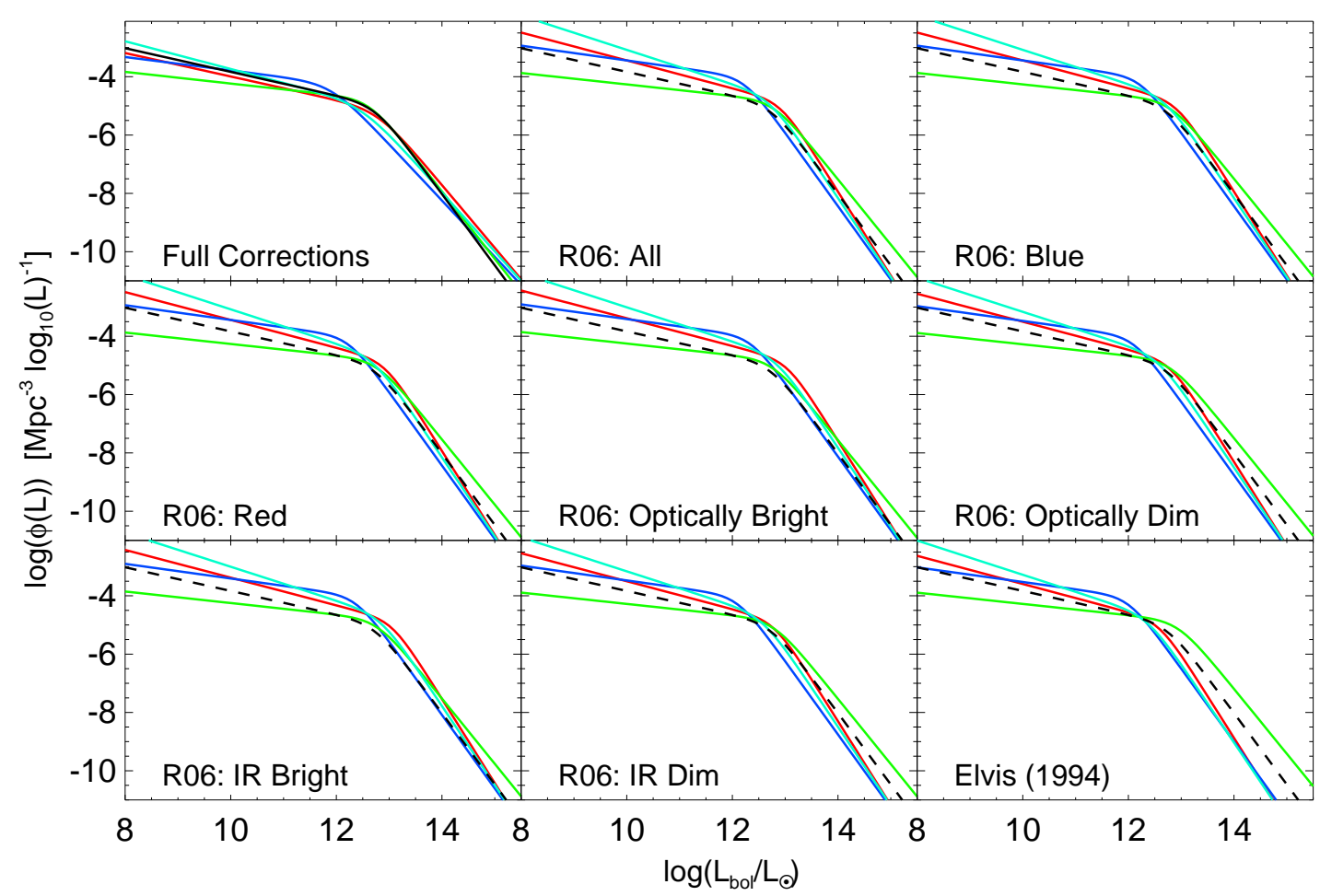

FIG. 3.- Comparison of the best-fit bolometric QLFs to the observations in Figure 2 at $z \sim 1$, fitted independently to the optical (green), soft X-ray (blue), hard X-ray (red), and IR (cyan) data sets. Upper left panel shows the result using our full (luminosity-dependent) bolometric corrections, with the black line the bolometric QLF from fitting to the data at all wavelengths simultaneously (reproduced as the dashed black line in the other panels), subsequent panels adopt constant (luminosity-independent) corrections from Richards et al. (2006c) based on the mean spectrum of the complete (all), blue, red, optically bright, optically dim, IR bright, and IR dim sub-samples considered therein. Lower right panel adopts the Elvis et al. (1994) mean spectrum. Accounting for the dependence of spectral shape on luminosity yields a consistent bolometric QLF across all frequencies and luminosities, whereas considering a single mean spectrum is generally appropriate only for narrow luminosity and color intervals.

moderate redshifts.

Likewise, although not shown in Figure 2 we find no evidence for any dependence of bolometric corrections on redshift, in agreement with a number of direct measurements of quasar SEDs (e.g., Elvis et al. 1994; Vanden Berk et al. 2001; Telfer et al. 2002; Vignali et al.|2003; Fan et al.|2003; Steffen et al. 2006; Richards et al. 2006c; Shemmer et al. 2006). If the variation of e.g. $\alpha_{\mathrm{ox}}$ were primarily a redshift as opposed to luminosity dependence, or if there were e.g. a strong dependence of X-ray photon index on redshift as suggested by Kuhn et al. (2001) and Bechtold et al. (2003), then our inferred bolometric QLFs from different bands would diverge with redshift. Instead, they appear to be self-consistent given the redshift-independent spectral model at all $z=0-6$. Given the limitations of present data, however, it is still possible that less well-constrained portions of the spectrum evolve differently. For example, the IR SED could, in principle, evolve differently with luminosity or redshift from the optical SED (but see Jiang et al. 2006b, who find similar near-IR SEDs at high redshift), and Maiolino et al. (2004) have suggested that extinction curves may evolve at $z \gtrsim 4-5$ (although their proposed extinction curves change the optical depth at the wavelengths of interest by $\lesssim 10 \%$ ). There is no strong evidence for such additional evolution in the samples we consider, but these caveats should be considered in any extrapolation of our fitting to less well-sampled frequencies and redshifts.

\subsection{The Relation of Bolometric to Observed QLFs}

We briefly examine the relation between the shape of the bolometric QLF and the observed QLF at different frequencies. Figure 4 shows the fit to the bolometric QLF from Figure 2, at $z \sim 1$, and the resulting observed QLF in different bands. Although our fitting considers all observations in the bands given in Table 1 we have renormalized all optical observations to the $B$-band for plotting purposes (likewise for the other bands). We plot all observations in the same units ( $B$-band and $15 \mu \mathrm{m}$ show $\nu L_{\nu}$ ) for the sake of direct comparison.

The optical/UV spectrum dominates the bolometric luminosity (at least in bright, unobscured quasars), and therefore the optical bolometric correction depends only weakly on luminosity. Consequently, the bright-end slope of the optical QLF is essentially identical to that of the bolometric QLF, offset by a nearly constant (when calibrated for optically bright quasars) bolometric correction. However, at the faint end, obscuration significantly flattens the optical QLF. In the hard $\mathrm{X}$-ray, this is reversed. The faint-end slope is relatively unaffected by obscuration and directly probes the bolometric faint-end, but the change in bolometric corrections (as well as the scatter in bolometric corrections at fixed $L$ ) alters the bright-end shape considerably. The IR, unsurprisingly, provides in many ways the most direct estimate of the bolometric luminosity distribution, but is the most poorly constrained by current observations. The soft X-ray, while yielding some of the tightest observational constraints and critical for evaluat- 
ing e.g. the UV contributions of quasars, is affected at the faint and bright ends by obscuration and changing bolometric corrections, respectively.

\subsection{The Contribution of Quasar Host Galaxy Light}

We have so far ignored the contribution of quasar host galaxies to their observed luminosities. Observed luminosity functions, however, do not generally remove this host contamination. In the X-ray bands, this is not of course expected to be a serious concern, and in the emission-line luminosity functions of Hao et al. (2005) we consider their more conservative AGN cut which should eliminate much of the contamination from star-forming nuclear regions in the hosts. However, this contribution may still be problematic at optical and IR wavelengths. If we knew the black hole masses of quasars in the observed QLFs, it would be straightforward to at least estimate an average host contribution or bias, as there is a well-established correlation between black hole mass and host galaxy optical luminosity (or mass) (e.g., Kormendy \& Richstone 1995). Lacking this information, we must adopt some estimate of the observed quasar Eddington ratio $\left(L / L_{\text {Edd }}\right)$ distribution to convert from a bolometric luminosity to black hole mass and corresponding host luminosity.

In Figure 5] we consider several simple, representative cases to estimate the possible effects of our neglecting host luminosities. We compare the $B$-band QLF neglecting host light (our fiducial model) and alternatively, assuming all quasars are radiating at the Eddington luminosity. This gives a black hole mass for each $L$, and we use the black hole masshost $B$-band luminosity relationship from Marconi \& Hunt (2003) to determine the corresponding host contribution (see also Vanden Berk et al. 2006, who measure a similar $L / L_{\text {Edd }^{-}}$ dependent relation directly in AGN). The quasar luminosities are corrected and attenuated as before, and then the (unattenuated) host luminosity is added.

Strictly speaking, this is not necessarily self-consistent, as the observational calibrations of bolometric corrections do not necessarily remove host galaxy contributions. However, our construction of the intrinsic quasar SED should effectively exclude host contributions (compare e.g. Richards et al. (2006c) who explicitly remove a rough lower limit to the host contribution), so long as there is not a substantial host contribution at $\sim 2500 \AA$ which would significantly affect the determination of $\alpha_{\mathrm{ox}}$ (although this is not expected at these frequencies, and the relation in Steffen et al. (2006) is calibrated from only Type 1 AGN). In any case, these effects are second-order to those shown in Figure 5

We also consider the case where all quasars radiate at an Eddington ratio $L / L_{\text {Edd }}=0.3$ and $L / L_{\text {Edd }}=0.1$. As an alternative simplification, we consider the case if the QLF is a pure Eddington ratio sequence with $L / L_{\text {Edd }}=L / L_{*}\left(L_{*}\right.$ is the fitted QLF break luminosity). It makes no difference if we allow $L / L_{\text {Edd }}>1$ or fix the maximum $L / L_{\text {Edd }}=1$, as the host contribution is minimal in either case. We show this for the $B$-band, but the effects in the mid-IR are similar (given e.g. the typical early-type spectrum of Fioc \& Rocca-Volmerange (1997); effects are also similar for other host types).

Figure 5demonstrates that including quasar host galaxy luminosities should be a small effect, except at the lowest luminosities $M_{B} \gtrsim-23$ (bolometric $L \lesssim 10^{12} L_{\odot}$ ). This is unsurprising, as at bright observed $M_{B}$, obscuration is small and Eddington ratios are expected to be high. In general, this implies a negligible impact on our subsequent calculations, as the deepest optical observations only just probe the range in which host luminosity contamination becomes significant (the larger baseline at low luminosities comes from hard X-ray observations, which do observe significant numbers of optically normal galaxies hosting low-luminosity Xray AGN, e.g. Barger et al. 2005). At higher redshift, flux limited samples are further removed from these luminosities, and Eddington ratios are expected to be uniformly high (at least in optically-selected samples, e.g. McLure \& Dunlop 2004). Moreover, observations of the relation between black hole mass and host luminosity at high redshift, albeit considerably uncertain, suggest that host luminosities either remain constant or decrease at a given $M_{\mathrm{BH}}$ (e.g., Peng et al. 2006).

However, this strongly cautions the interpretation of deeper optical, near- or mid-IR surveys, especially at low redshifts $z \lesssim 0.5$, where Eddington ratios may well be low $(\lesssim 0.1)$. If such surveys seek to probe luminosities $\lesssim 10^{12} L_{\odot}$, careful consideration of the contribution from quasar hosts is necessary to determine the actual quasar contribution at low luminosities. For example, Figure 5] demonstrates that the observed faint-end QLF slope in these bands can be significantly biased by host contamination at magnitudes lower than those currently probed, in a manner sensitive to the Eddington ratio or black hole mass distribution. These effects will be even more pronounced in the near-IR, as the ratio of host to quasar luminosity in unobscured objects has a typical maximum at $\nu \sim 1.6 \mu \mathrm{m}$ (see e.g. Figure 11 of Richards et al. 2006c).

\section{THE BOLOMETRIC QLF}

\subsection{The Bolometric QLF at Specific Redshifts}

We combine the binned QLF measurements to examine the QLF "at" a single redshift. Of course, the observations are not made over identical redshift intervals, so we re-normalize each to the same redshift (as $n_{\mathrm{obs}} / n_{\mathrm{mdl}}$ ) with the best-fit analytic QLF fit from the same (or appropriate companion) paper. This introduces some additional model dependence, so these fits should be considered as heuristic, but they will inform our subsequent choice of functional forms for more properly fitting to the redshift dependence of the bolometric QLF.

We follow standard practice and fit the QLF to a double power law

$$
\phi(L) \equiv \frac{\mathrm{d} \Phi}{\mathrm{d} \log (L)}=\frac{\phi_{*}}{\left(L / L_{*}\right)^{\gamma_{1}}+\left(L / L_{*}\right)^{\gamma_{2}}},
$$

with normalization $\phi_{*}$, break luminosity $L_{*}$, faint-end slope $\gamma_{1}$, and bright-end slope $\gamma_{2}$. Note that conventions for the double power law are often different in optical and X-ray analyses; for optical QLFs typically the double power law is defined in terms of (e.g., Peterson 1997; Croom et al. 2004)

$$
\frac{\mathrm{d} \Phi}{\mathrm{d} L}=\frac{\phi_{*}^{\prime} / L_{*}}{\left(L / L_{*}\right)^{-\alpha}+\left(L / L_{*}\right)^{-\beta}},
$$

or per unit absolute magnitude

$$
\frac{\mathrm{d} \Phi}{\mathrm{d} M}=\frac{\phi_{*}^{\prime \prime}}{10^{0.4(\alpha+1)\left(M-M_{*}\right)}+10^{0.4(\beta+1)\left(M-M_{*}\right)}},
$$

which in our notation gives $\alpha=-\left(\gamma_{1}+1\right), \beta=-\left(\gamma_{2}+1\right), \phi_{*}^{\prime}=$ $\phi_{*} / \ln 10$, and $\phi_{*}^{\prime \prime}=0.4 \phi_{*}$.

The resulting bolometric QLF at several redshifts is shown in Figures 6 \& 7 At each redshift, all binned observations from Table 1 in overlapping redshift intervals are shown. The QLFs from optical, soft and hard X-ray, IR, and emission-line measurements yield a self-consistent bolometric QLF at all luminosities and redshifts probed, when a full modeling of the 


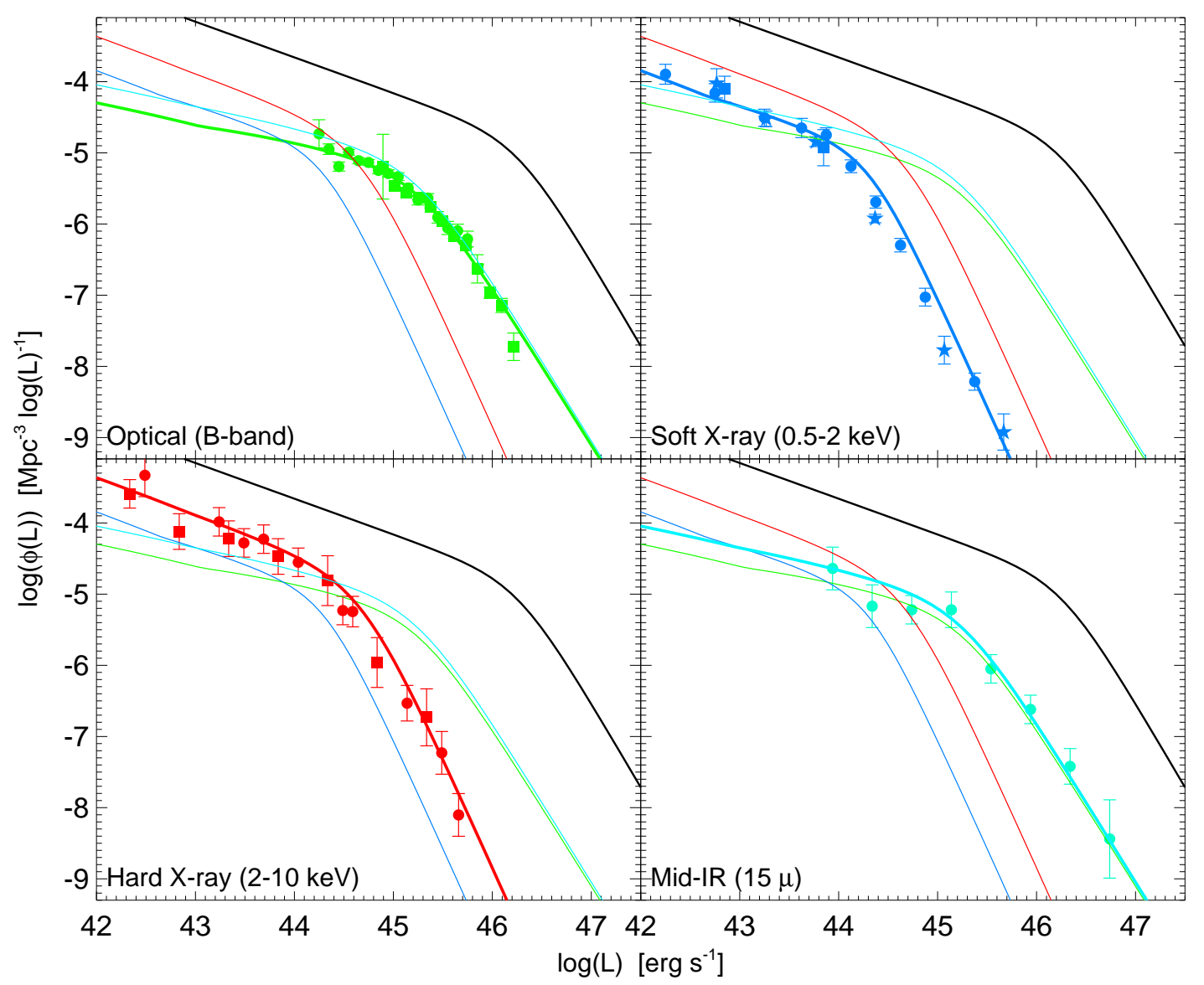

FIG. 4.- The best-fit bolometric QLF at $z \sim 1$ (black lines), with the resulting observed QLF in each of several bands: optical (green), soft X-ray (blue), hard X-ray (red), and IR (cyan). Each corresponding panel plots the compiled observations in Table 1 for the appropriate redshift and frequency (points). Symbols denote the parent sample as given in Table 1 The observations at each band are consistently produced from a single bolometric QLF, and together provide strong constraints on the QLF shape. Different wavebands accurately represent different aspects of the bolometric QLF shape. With only a weakly $L$-dependent bolometric correction but significant effects of obscuration, the optical QLF faithfully traces the bolometric bright end shape, but is flatter at faint $L$. Hard X-rays, conversely, are weakly affected by obscuration but can have a strongly luminosity-dependent bolometric correction, reproducing the faint end shape but being steeper at bright $L$. The IR QLF better follows the bolometric shape, but is currently least well-constrained.

observed spectral and $N_{\mathrm{H}}$ distributions is employed. Furthermore, the combination of this number of observations provides a large luminosity and redshift baseline, sampling the the faint end, break, and bright-end slope to redshifts $z \sim 4-5$.

We plot the best-fit parameters from these fits at a number of redshifts in Figure 8 (error bars show formal $1 \sigma$ uncertainties from the fits), and list them at several redshifts in Table 2 The normalization $\phi_{*}$ is roughly constant, while the break luminosity (which is quite tightly constrained for most of the redshift range) evolves by $\sim 2$ orders of magnitude. Note that the points where $\phi_{*}$ appears to deviate from being constant also have discrepant $L_{*}$ values — the degeneracy between the two is such that the value of $\phi_{*}$ is consistent with being constant at all $z$ for smooth evolution in $L_{*}$. There is an indication of evolution in both the faint-end and bright-end slopes, which we discuss below. The integrated bolometric luminosity density is well constrained, with the largest uncertainties only $\sim 0.15$ dex. We determine the luminosity density by integrating the best-fit luminosity function to $L=0$. At most redshifts $z \gtrsim 0.5$ the faint-end slope is relatively shallow, and choosing instead a cutoff at e.g. $L=10^{8}-10^{9} L_{\odot}$ changes the integrated luminosity density by $\lesssim 10 \%$. At the lowest redshifts, however, the faint-end slope is steep, and at $z=0$ where this is most pronounced, the luminosity density is $\sim 15 \%$ lower if we truncate at $L=10^{8} L_{\odot}\left(\sim 25 \%\right.$ lower for $\left.L=10^{9} L_{\odot}\right)$. This sensitivity to the steep faint-end slope at low- $z$ is the reason for the relatively large uncertainty in the luminosity density at low redshift. At high- $z$ the uncertainty owes to the limited amount of data. In any case there is a well-defined peak in the luminosity density at $z=2.154 \pm 0.052$ (formal error from fit; we expect a systematic error \pm 0.15 from choices in sampling and binning the observations), well outside the range where either of these systematic concerns is problematic.

\subsection{Analytic Fits as a Function of Redshift}

We characterize the QLF as a function of redshift by adopting a standard pure luminosity evolution (PLE) model, where the bolometric QLF is a double power law at all $z$, with constant $\gamma_{1}, \gamma_{2}$, and $\phi_{*}$, but an evolving $L_{*}$. We allow $L_{*}$ to evolve as a cubic polynomial in redshift,

$$
\log L_{*}=\left(\log L_{*}\right)_{0}+k_{L, 1} \xi+k_{L, 2} \xi^{2}+k_{L, 3} \xi^{3},
$$

where

$$
\xi=\log \left(\frac{1+z}{1+z_{\text {ref }}}\right) .
$$

Here, $k_{L, 1}, k_{L, 2}$, and $k_{L, 3}$ are free parameters, and we set $z_{\text {ref }}=2$ (which roughly minimizes the covariance in the fit). 


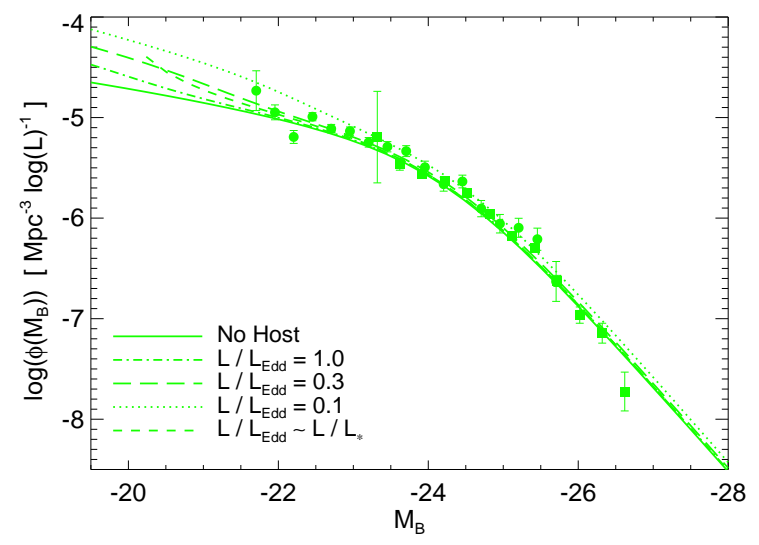

FIG. 5.- The observed $B$-band QLF (points) at $z \sim 1$, and that determined from the best-fit bolometric QLF in Figure 4 (solid line) ignoring contributions to the observed luminosity from quasar host galaxies. Also shown is the $B$-band QLF determined from the same bolometric QLF, but including the expected host galaxy contribution to the observed luminosities (normalized for a given Eddington ratio with the observed relation between black hole mass and host galaxy $B$-band luminosity from Marconi \& Hunt (2003)), assuming all quasars have constant $L / L_{\mathrm{Edd}}=1.0,0.3,0.1$ (dot-dashed, long-dashed, dotted lines, respectively), or assuming $L / L_{\mathrm{Edd}}=L / L_{*}$ (short dashed line; $L_{*}$ is the QLF break luminosity). Host galaxy contributions should be negligible at most luminosities and redshifts for the observed bands we consider, but will be important for future optical, near- and mid-IR surveys which probe fainter luminosities $L \lesssim 10^{12} L_{\odot}\left(M_{B} \gtrsim-23\right)$.

The cubic term is demanded by the data $\left(\Delta \chi^{2} \sim 600\right.$ on its addition), but higher order terms in $\xi$ are not $\left(\Delta \chi^{2} \lesssim 1\right)$. Since this model includes the evolution with redshift, we can simultaneously fit to all the data sets in Table 1 each over the appropriate redshift intervals of the observed samples.

The best-fit PLE model parameters are given in Table 3 and plotted as a function of redshift in Figure 8 and the resulting QLF is shown in Figures 6 \& 7 Although this provides a reasonable lowest-order approximation to the data, it fails at the faint end, underpredicting the abundance of low-luminosity sources at $z \lesssim 0.3$ and overpredicting it at $z \gtrsim 2$, and the fit is poor at $z \gtrsim 5$ with much too steep a bright end slope. Over the entire data set, the fit is unacceptable, with $\chi^{2} \approx 1924$ for $\nu=510$ degrees of freedom. A pure density evolution (PDE) model fares even worse, with $\chi^{2} / \nu=3255 / 510$ (although for completeness we provide the best-fit PDE parameters in Table 4h, unsurprising given that nearly every observed data set which resolves the break in the QLF favors the PLE form (e.g., Bovle et al.|2000; Mivaii et al.|2000; Ueda et al. 2003; Croom et al. 2004; Richards et al. 2005).

As discussed in $\S 1$ many recent studies have found evidence for evolution in the faint end beyond that predicted by PLE, with the density of lower-luminosity sources peaking at lower redshift than the density of higher-luminosity sources, and have fit this trend with a luminosity-dependent density evolution (LDDE) model (Schmidt \& Green 1983), while high-redshift samples have suggested evolution in the bright-end QLF shape, confirmed robustly as a flattening in $\gamma_{2}$ for the first time in a homogeneous sample by the SDSS (Richards et al. 2006c). We wish to incorporate this additional evolution into our model.

For comparison with these results and future X-ray surveys, we fit to an LDDE form allowing maximal flexibility of the parameters,

$$
\phi(L, z)=\phi(L, 0) e_{\mathrm{d}}(L, z)
$$

$$
=\frac{\phi_{*}}{\left(L / L_{*}\right)^{\gamma_{1}}+\left(L / L_{*}\right)^{\gamma_{2}}} e_{\mathrm{d}}(L, z) .
$$

The density function $e_{\mathrm{d}}$ is given by

$$
e_{\mathrm{d}}(L, z)= \begin{cases}(1+z)^{p 1} & \left(z \leq z_{c}\right) \\ \left(1+z_{c}\right)^{p 1}\left[(1+z) /\left(1+z_{c}\right)\right]^{p 2} & \left(z>z_{c}\right)\end{cases}
$$

with

$$
z_{c}(L)= \begin{cases}z_{c, 0}\left(L / L_{c}\right)^{\alpha} & \left(L \leq L_{c}\right) \\ z_{c, 0} & \left(L>L_{c}\right)\end{cases}
$$

and

$$
\begin{aligned}
& p 1(L)=p 1_{46}+\beta_{1}\left[\log \left(L / 10^{46} \operatorname{erg~s}^{-1}\right)\right] \\
& p 2(L)=p 2_{46}+\beta_{2}\left[\log \left(L / 10^{46} \operatorname{erg~s}^{-1}\right)\right] .
\end{aligned}
$$

Note that some authors (e.g., Hasinger et al. 2005) adopt an alternative normalization convention in terms of $A_{44} \equiv \phi(L=$ $\left.10^{44} \mathrm{erg} \mathrm{s}^{-1}, z=z_{c}\right)$ and $z_{c, 44} \equiv z_{c}\left(L=10^{44} \mathrm{erg} \mathrm{s}^{-1}\right)$, but these choices are not particularly convenient for the bolometric QLF. The 11 free parameters in this fit are then $L_{*}, \phi_{*}, \gamma_{1}$, $\gamma_{2}, z_{c, 0}, L_{c}, \alpha, p 1_{46}, p 2_{46}, \beta_{1}, \beta_{2}$. Their best-fit values are given in Table 4

The LDDE form can effectively describe evolution in the faint-end QLF slope, but it does not allow for evolution in the bright-end slope. It also has a tendency to introduce a "second break" in the faint end of the QLF (i.e. if the faint end flattens there is often some $L$ below which it rises steeply again), for which we see no evidence. Ultimately, the improvement over the PLE fit is highly significant, with $\chi^{2} / \nu=1389 / 507$, but there is still room for substantial improvement.

Therefore, we instead consider the PLE form above, but allow both the bright- and faint-end slopes to evolve with redshift. For the faint-end slope, using the fitted points at each $z$ to inform our choice of functional form, we model $\gamma_{1}$ as a power-law in redshift,

$$
\begin{aligned}
\gamma_{1} & =\left(\gamma_{1}\right)_{0} \times 10^{k \gamma_{1}} \xi \\
= & \left(\gamma_{1}\right)_{0}\left(\frac{1+z}{1+z_{\text {ref }}}\right)^{k \gamma_{1}},
\end{aligned}
$$

where again $z_{\text {ref }}=2$ is fixed. Allowing this dependence (while still holding $\gamma_{2}$ constant) significantly improves the quality of the fit relative to the PLE model, reducing $\chi^{2}$ by $\Delta \chi^{2} \approx 500$ with the addition of one parameter, to $\chi^{2} / \nu=1422 / 510$. The values for this fit are given in Table 3 There is no evidence for higher-order terms $\left(\Delta \chi^{2} \lesssim 1\right.$ for the addition of a secondorder power in $\xi$ ). We have also tested different functional forms, and do not find any which provide a significantly better fit. This choice also has the advantage that it extrapolates to a flat $\gamma_{1} \rightarrow 0$ (as opposed to a negative, likely unphysical) slope at high redshift.

Parameterizing evolution in the bright-end slope is more difficult. There appears to be evidence for a steepening of the bright-end slope (increase in $\gamma_{2}$ ) from $z \sim 0$ to $z \sim 1.5$, then a flattening with redshift. We find the best results for a double-power law of the form

$$
\begin{aligned}
\gamma_{2} & =\left(\gamma_{2}\right)_{0} \times 2\left[10^{k_{\gamma_{2}, 1} \xi}+10^{k_{\gamma_{2}, 2} \xi}\right]^{-1} \\
& =\frac{2\left(\gamma_{2}\right)_{0}}{\left(\frac{1+z}{1+z_{\text {ref }}}\right)^{k_{\gamma_{2}, 1}}+\left(\frac{1+z}{1+z_{\text {ref }}}\right)^{k_{\gamma_{2}, 2}}} .
\end{aligned}
$$

Here, $k_{\gamma_{2}, 1}$ describes the rise of $\gamma_{2}$ with $z$ at low redshift, and $k_{\gamma_{2}, 2}$ describes the fall of $\gamma_{2}$ with $z$ at high redshift. The fac- 


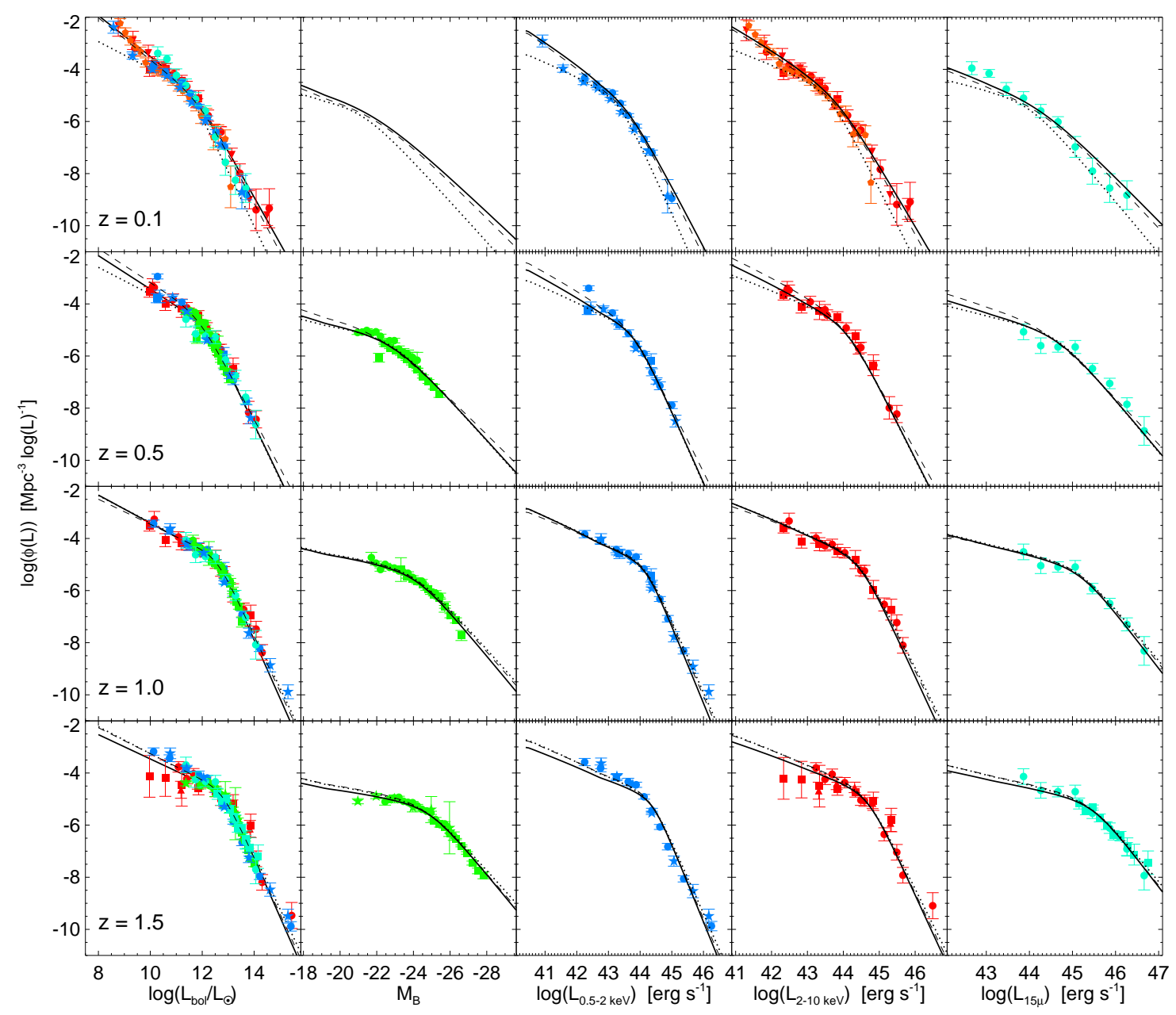

FIG. 6. - The best-fit bolometric QLF at each of several redshifts (left panels; shown as $n_{\mathrm{mdl}} / n_{\mathrm{obs}}$ ), and the corresponding observed QLF in $B$-band (center-left; green), soft X-rays $(0.5-2 \mathrm{keV})$ (center; blue), hard X-rays $(2-10 \mathrm{keV})$ (center-right; red), and mid-IR $(15 \mu \mathrm{m})$ (right; cyan). Rather than add a series of panels for a single data set, the emission-line luminosity functions of Hao et al. (2005) are shown (orange) in the $z=0.1$ hard X-ray panel (rescaled by $n_{\mathrm{obs}} / n_{\mathrm{mdl}}$, but equivalently directly converted to hard X-ray luminosities following Heckman et al. 2005). Lines show the best-fit evolving double power-law model to all redshifts (solid), the best-fit model at the given redshift (dashed), and the best-fit PLE model (dotted). Points shown are the compiled observations from Table 1 with the plotting symbols for each observed sample listed therein.

tor of 2 is inserted such that if $k_{\gamma_{2}, 1}=k_{\gamma_{2}, 2}=0$, the resulting $\gamma_{2}=\left(\gamma_{2}\right)_{0}$. The best-fit parameters, fixing $\gamma_{1}$ or allowing both slopes to evolve simultaneously, are given in Table 3 We find that $k_{\gamma_{2}, 1}$ is non-zero at high formal significance, but cannot say whether this steepening up to $z \sim 1.5$ is "real" in any robust sense; however, including this parameter does significantly improve the accuracy of our fitting function in representing the binned data. The flattening at high redshifts is more significant $(\sim 7 \sigma)$. Including these two parameters greatly improves the quality of the fit by $\Delta \chi^{2} \approx 600$, giving $\chi^{2} / \nu=1312 / 509$ for a fit with constant $\gamma_{1}$ or $\chi^{2} / \nu=1007 / 508$ for a simultaneous fit including evolution in $\gamma_{1}$ and $\gamma_{2}$. Again, there is no evidence for terms describing further evolution. Although our final best fit does not cross this limit until the highest redshifts, we also formally enforce a lower limit $\gamma_{2} \geq 1.3$ (to prevent an unphysical divergence in the luminosity density).

The best-fit parameters as a function of redshift (allowing all free parameters to vary) are plotted in Figure 8 There is a slight offset between the $L_{*}$ and $\phi_{*}$ of this fit and that fitted at specific redshifts, but this owes to their covariance.
This can be seen in the bolometric luminosity density, which accurately traces the fit predictions (note that the PLE model significantly overpredicts the luminosity density at $z>2$, as it overpredicts the number of faint sources). Our restriction $\gamma_{2} \geq 1.3$ is important in extrapolating beyond $z \sim 5$ to prevent a divergence in the number and luminosity density of bright sources. We also plot the predicted number density of quasars with $M_{B}<-27$ as a function of redshift, which agrees with the direct observations (expected, but nevertheless a reassuring consistency check).

Figure 9 plots the number density of quasars integrated over various luminosity intervals, in various bands as a function of redshift, from the best-fit model and the compiled observations in Table 1 Although this information is contained in Figures 6 and 7 Figure 9 nicely illustrates an essential trend captured by the LDDE or evolving double power-law forms, namely that the density of lower-luminosity sources peaks at lower redshift than that of higher-luminosity sources. The trend is evident in all bands we consider.

We briefly consider an alternate model for the shape of the QLF, which illustrates several important points. Instead of 


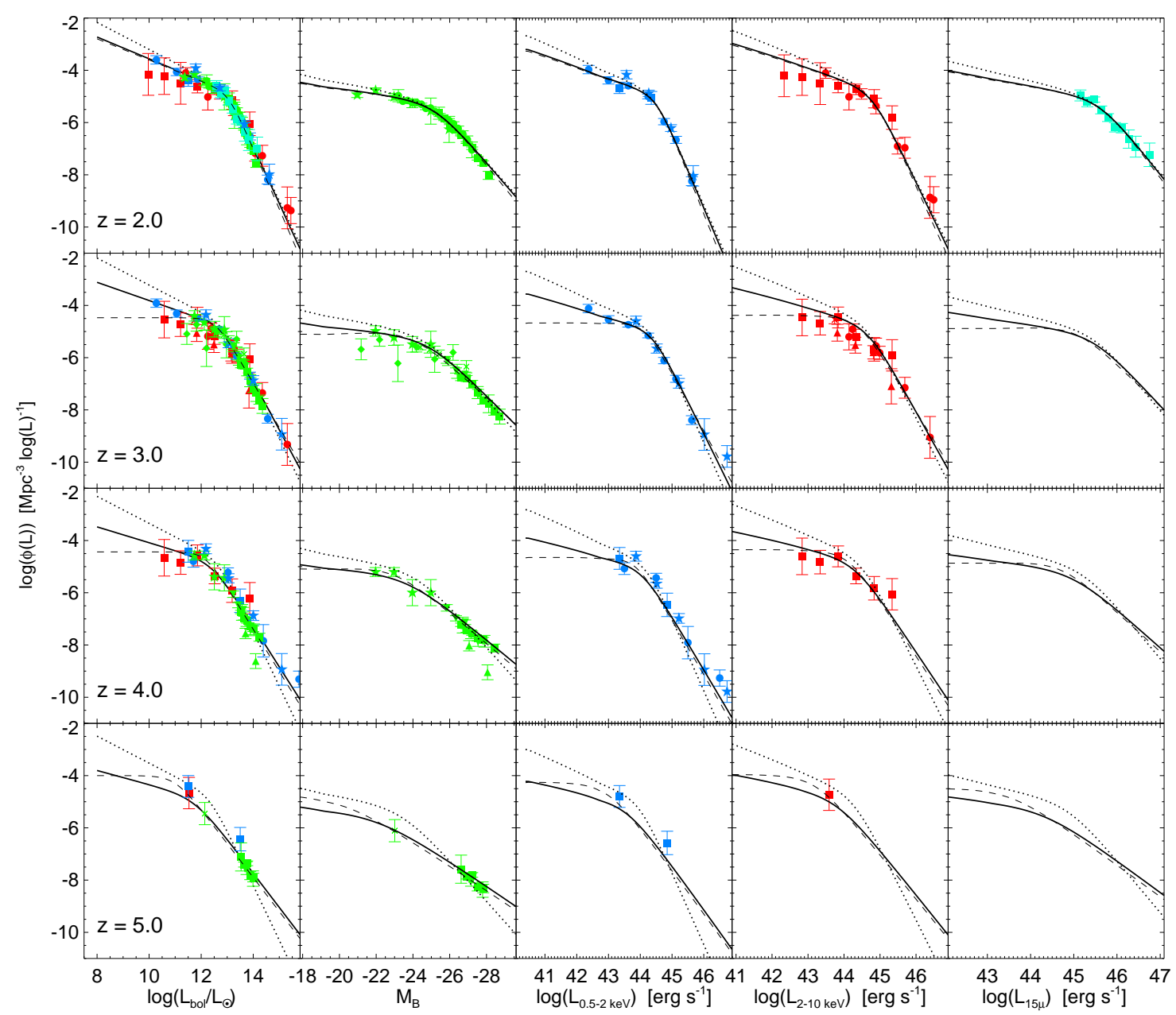

FIG. 7.- As Figure 6 but at higher redshifts, as labeled.

the double power-law of Equation [6, we consider a modified Schechter function

$$
\phi(L)=\phi_{*}\left(L / L_{*}\right)^{-\gamma_{1}} \exp \left\{-\left(\frac{L}{L_{*}}\right)^{\gamma_{2}}\right\} .
$$

We have also, for example, adopted polynomials of arbitrarily high order (although the fits typically do not improve beyond fourth order). In either case, the fit is quite similar at most luminosities (with similar $\chi^{2} / \nu$ ), implying that there is no dramatic shape dependence which we have not captured. However, such a fit does exhibit smoother curvature rather than a sharp break at $L_{*}$, evidence for which has been seen in some optical samples (e.g., Wolf et al. 2003; Richards et al. 2005). At the highest luminosities ( $\gtrsim 1$ dex above $L_{*}$, typically $\left.L_{\text {bol }} \gtrsim 10^{14} L_{\odot}\right)$ the implied number of quasars is an order of magnitude lower for these parameterizations than for the double power law prediction, and falls much more rapidly. The resulting observed luminosity functions are more sensitive to the estimated dispersion in bolometric corrections, but in either case the highest luminosity soft and hard X-ray objects in Figures [ [ \& 7] are substantially affected by quasars shifting into slightly larger bins of soft or hard X-ray luminosity owing to different spectral shapes (e.g. the scatter in $\left.\alpha_{\text {ox }}\right)$. It is important to account for this effect when attempting to infer the number density of the most massive black holes and most luminous quasars, as a naive extrapolation of the median bolometric corrections applied to the most X-ray bright quasars implies extreme (and potentially unphysical) bolometric luminosities $\gtrsim 10^{15} L_{\odot}$ (i.e. a $\gtrsim 3 \times 10^{10} M_{\odot}$ black hole at the Eddington rate). Multiwavelength observations of these particular objects and further study from large area surveys which do not have to bin in widely spaced luminosity intervals will be critical in breaking the degeneracies between these fits to the intrinsic bolometric QLF and the double power-law form.

We are unable to find any further dependences which significantly improve the best-fit QLF. Allowing the parameters describing the observed column density distribution and spectral shape, and their respective luminosity dependence, to vary simultaneously, yields only marginal improvement. It appears that the remaining scatter in the data does not mostly owe to a failure to capture some remaining dependence. In Table 1 we list the $\chi^{2} / \nu$ for each sample with respect to the best-fit full model of the evolution of the QLF in Table 3 The agreement with most samples is good, and the largest, most wellconstrained samples (with small typical $\ll 0.1$ dex errorbars) give $\chi^{2} / \nu \lesssim 3$, in each case comparable to or smaller than the reduced $\chi^{2}$ found by the respective authors in fits to those individual data sets. To the extent that the functional forms adopted by these authors cannot reduce the variance below 

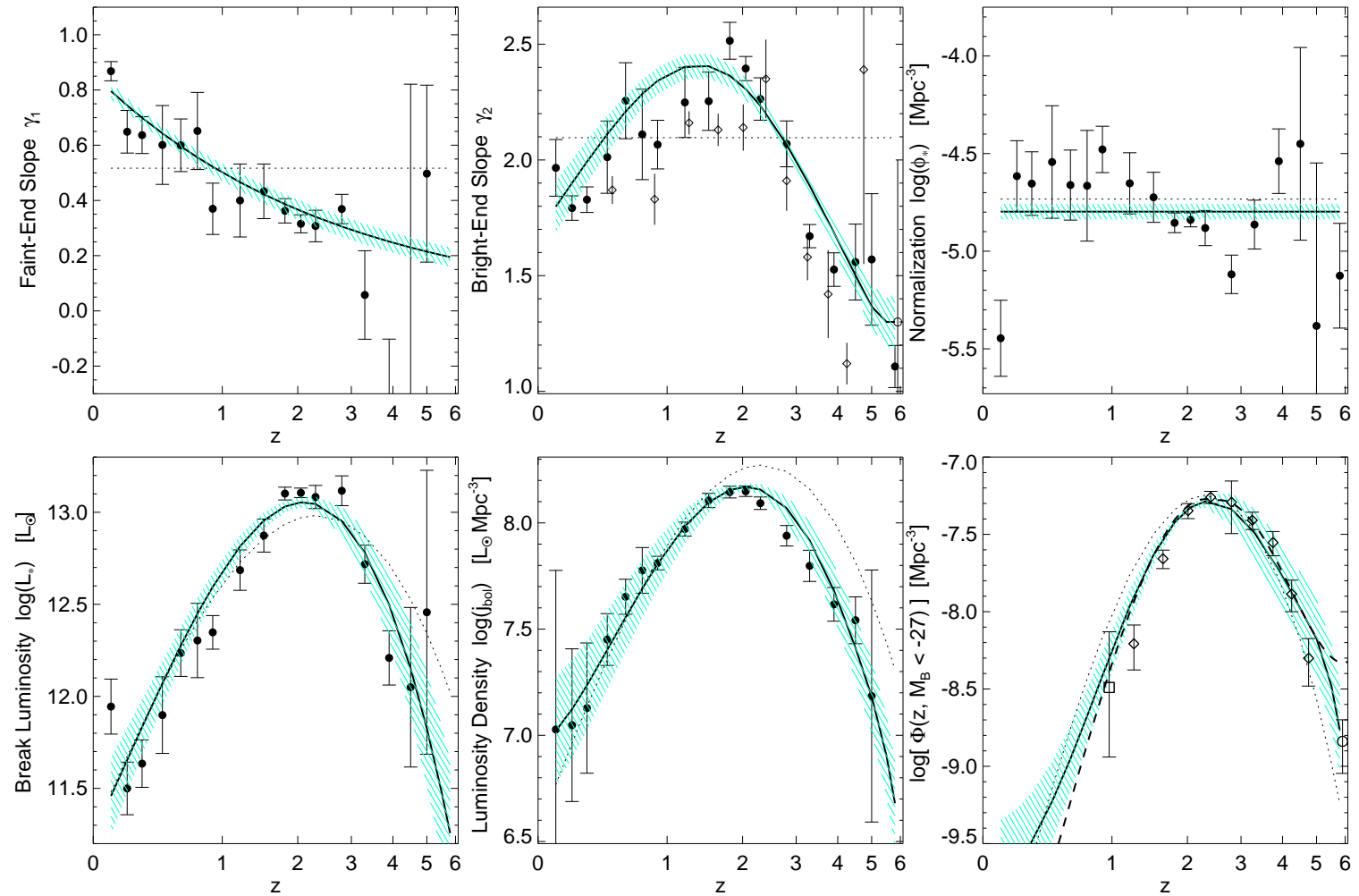

FIG. 8. - The best-fit QLF double-power law parameters as a function of redshift. Points show the best-fit values to data at each redshift, dotted lines the best-fit PLE model, and solid lines the best-fit full model (with cyan shaded range showing the $1 \sigma$ uncertainty). Open diamonds in $\gamma_{2}(z)$ show the bright-end slope fits from Richards et al. (2006b). Although PLE is appropriate for a lowest-order fit, both the bright and faint-end slopes evolve with redshift to high significance $(>6 \sigma)$. Lower right shows the predicted number density of bright optical $\left(M_{B}<-27\right)$ quasars from the full fit (solid), compared to that observed in Croom et al. (2004) (square), Richards et al. (2006b) (diamonds and dashed line), and Fan et al. (2004) (circle).

this level, we would not expect to do better in the combined sample.

There will also be some unavoidable variance introduced owing to systematic variations between independent data sets. Various observational calibrations, the model dependences inherent in calculating a binned QLF, and most of all cosmic variance will all contribute to sample-to-sample differences. In fact, we find that allowing for even a small $\sim 0.05 \mathrm{dex}$ $(10-15 \%)$ systematic normalization variance between samples, most of the remaining scatter is accounted for, with a best-fit model improvement $\Delta \chi^{2} \sim 500$. We provide the values of this fit in Table 3 but caution that we have increased the sample-to-sample variance by this amount uniformly, when in fact systematic effects such as cosmic variance will be smaller for large surveys such as the SDSS and 2dF than for the small deep fields of Chandra and XMM. Consequently, this underweights some of the most well-constrained observations, and the fit results should be considered to be heuristic.

\section{THE BUILDUP OF THE BLACK HOLE POPULATION}

\subsection{Model-Dependent Quantities}

We can gain further insight into the evolution of the QLF, albeit at the cost of some model dependence, by deconvolving the observed quasar luminosity function with a theoretical model for the quasar light curve or lifetime. This method is well-established (e.g., Salucci et al. 1999; Yu \& Tremaine 2002; Marconi et al. 2004; Shankar et al. 2004: Yu \& Lu 2004), but most studies adopt simplified "toy" light curves with accretion at a constant (fitted) absolute rate or Eddington ratio and fitted lifetimes and duty cycles. We instead follow Hopkins et al. (2006b), who derive physically motivated quasar light curves from simulations of merging galaxies that include black hole growth (e.g. Springel et al. 2005b) and which are consistent with a large range of observational constraints that cannot be reproduced by idealized models. This also removes the various fitting degeneracies - for a given bolometric QLF, the quasar light curves determined in simulations yield a unique black hole mass function, cosmic X-ray background spectrum, and self-consistent black hole and host galaxy properties.

Given a consistent model of the quasar lifetime/lightcurve, the observed bolometric QLF is given by the convolution of the rate of quasar formation or "triggering" with the differential quasar lifetime,

$$
\phi(L)=\int \dot{\phi}\left(M_{B H}\right) \frac{\mathrm{d} t}{\mathrm{~d} \log L}\left(L \mid M_{B H}\right) \mathrm{d} \log M_{B H}
$$

where

$$
\dot{\phi}\left(M_{B H}\right)=\frac{\mathrm{d} \Phi\left(M_{B H}, t\right)}{\mathrm{d} \log M_{B H} \mathrm{~d} t}
$$

is the rate of formation of black holes of a relic mass $M_{B H}$ at cosmic time $t$. Since $\mathrm{d} t / \mathrm{d} \log L$ is completely determined in the simulations and analytical models of Hopkins et al. (2006a b), we can fit to $\dot{\phi}\left(M_{B H}\right)$ in the same manner that we have fitted $\phi(L)$.

We assume a double power-law form for $\dot{\phi}\left(M_{\mathrm{BH}}\right)$ at all $z$,

$$
\dot{\phi}\left(M_{\mathrm{BH}}\right)=\frac{\dot{\phi}_{*}}{\left(M_{\mathrm{BH}} / M_{*}\right)^{\eta_{1}}+\left(M_{\mathrm{BH}} / M_{*}\right)^{\eta_{2}}}
$$



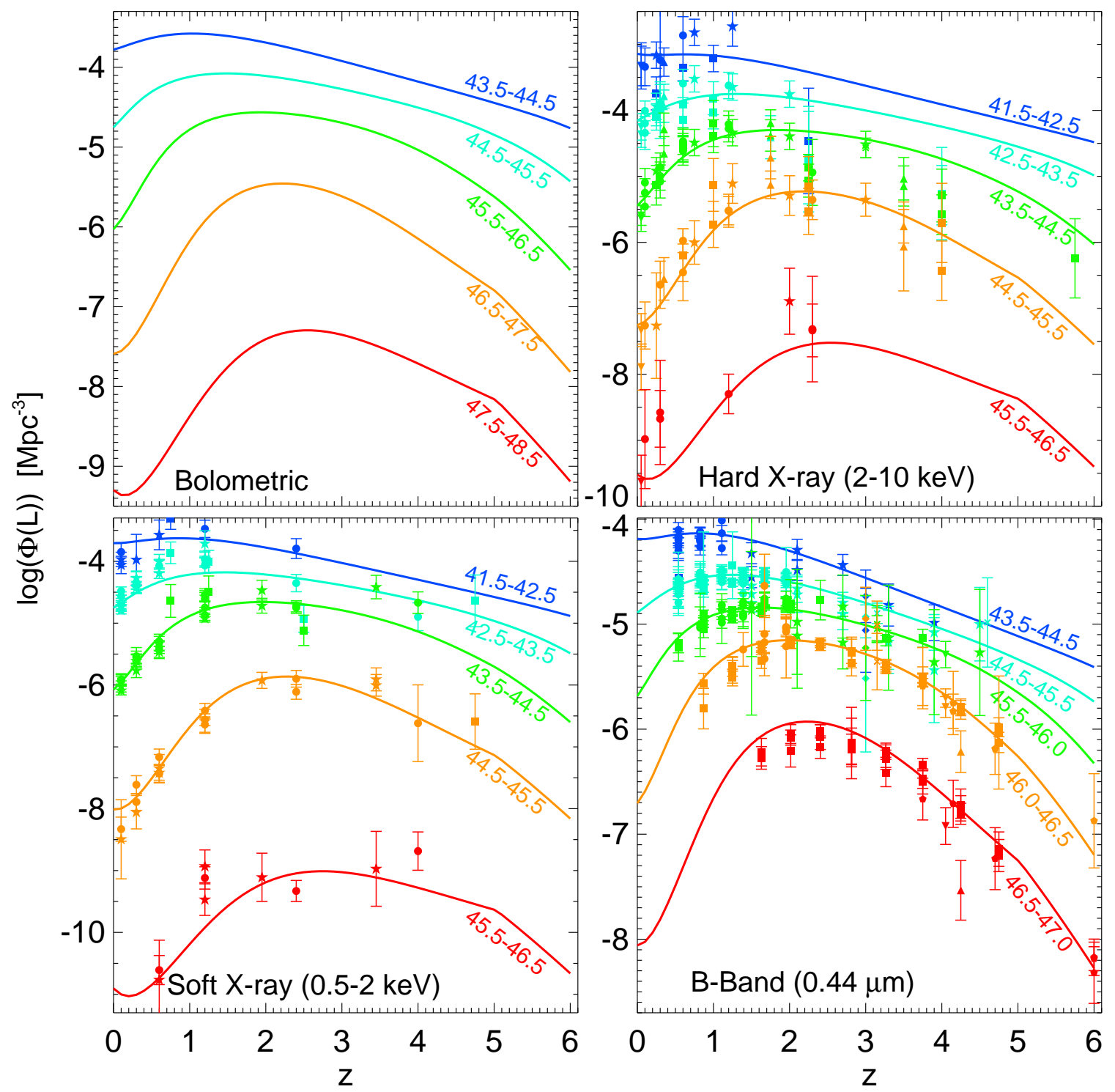

FIG. 9.- Total number density of quasars in various luminosity intervals (in $\log \left(L_{\text {band }} /\left[\mathrm{erg} \mathrm{s}^{-1}\right]\right)$ as labeled) as a function of redshift, from the best-fit evolving double-power law model (lines) and the compiled observations in Table1 (points of corresponding colors, symbols for each sample listed therein) in bolometric luminosity, $B$-band, soft X-rays $(0.5-2 \mathrm{keV})$, and hard X-rays $(2-10 \mathrm{keV})$. The trend that the density of lower-luminosity AGN peaks at lower redshift is manifest in all bands.

with normalization $\dot{\phi}_{*}$, break $M_{*}$, and faint-end and brightend slopes $\eta_{1}$ and $\eta_{2}$, respectively. We determine an analytical fit to $\dot{\phi}\left(M_{\mathrm{BH}}, z\right)$ in the same manner as $\phi(L, z)$. We allow $\log M_{*}$ to vary as a cubic in $\xi$ just as $L_{*}$, and allow the highmass slope $\eta_{2}$ to vary just as $\gamma_{2}$, as well. There is no evidence for evolution of the low-mass slope $\eta_{1}$. In this formulation there is evidence for evolution in $\dot{\phi}_{*}$ above $z \sim 2$, so we fix it to a constant below $z_{\text {ref }} \equiv 2$, and allow it to evolve as a powerlaw above $z_{\text {ref }}$,

$$
\dot{\phi}_{*}(z)= \begin{cases}\left(\dot{\phi}_{*}\right)_{0} & \left(z \leq z_{\mathrm{ref}}\right) \\ \left(\dot{\phi}_{*}\right)_{0}\left[(1+z) /\left(1+z_{\mathrm{ref}}\right)\right]^{k_{\dot{\phi}}} & \left(z>z_{\mathrm{ref}}\right) .\end{cases}
$$

In Figure 10 we plot the best-fit normalization $\dot{\phi}_{*}$ and break (characteristic mass in formation) $M_{*}$ as a function of redshift. At high redshifts, objects build up rapidly, until $z \sim 2$, after which the rate of merger/black hole growth "events" flattens, and activity ceases in the most massive systems and rapidly moves to less massive objects at lower redshifts, perhaps driven by feedback mechanisms quenching activity in the higher-mass systems. Given the adopted lifetime models, the observed faint-end $\phi(L)$ slope $\gamma_{1}$ is dominated by sources with $L \ll L_{\mathrm{Edd}}\left(M_{\mathrm{BH}}\right)$ and is determined by the quasar lifetime as a function of $M_{\mathrm{BH}}$. Figure 10 shows the predicted $\gamma_{1}(z)$ from the quasar lifetime model given the best-fit $\dot{\phi}\left(M_{\mathrm{BH}}\right)$ at each $z$, compared to the direct fits from Figure 8 the agreement is good despite $\eta_{1}$ being nearly constant and much flatter $\eta_{1} \approx 0.0-0.2$ at all redshifts (see also Figure 3 of Hopkins et al. (2006a)).

\subsection{Integrated Quantities}

If all black holes accrete with some constant radiative efficiency $\epsilon_{r}$,

$$
L_{\mathrm{bol}}=\epsilon_{r} \dot{M} c^{2}
$$



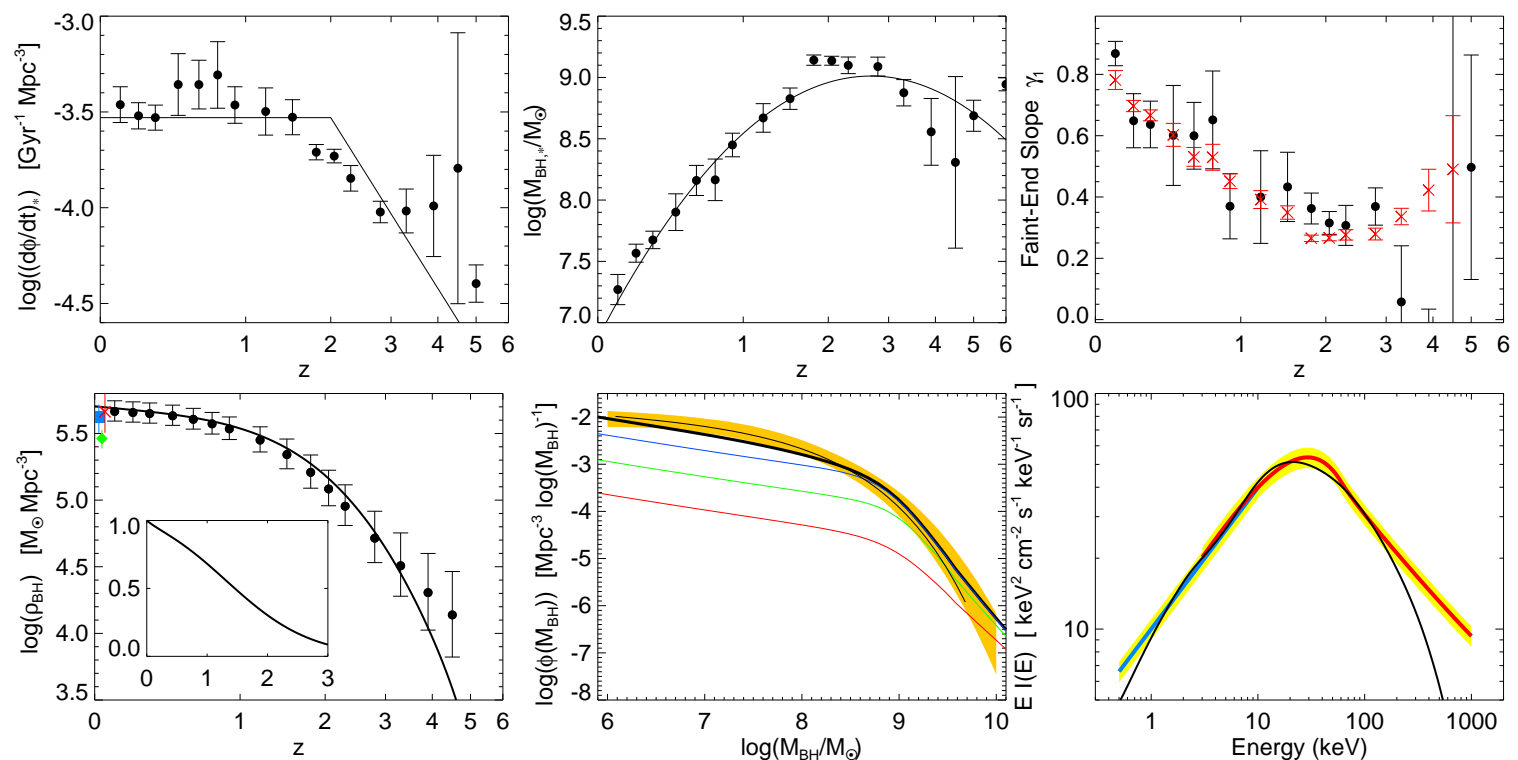

FIG. 10.- Best-fit parameters to $\dot{\phi}\left(M_{\mathrm{BH}}\right)$, the "birthrate" or formation rate of black holes at a given redshift, given the quasar lifetimes determined in the simulations of Hopkins et al. (2006a b). Top left shows $d \phi / d t$, the quasar activation / birthrate normalization. Top center shows $M_{*}$, the break in the characteristic final black hole mass forming at a given $z$. Points are fitted at each redshift, solid lines show best-fit from $\dot{\phi}\left(M_{\mathrm{BH}}\right)$ as a function of redshift. Top right shows the faint-end slope of the observed QLF from Figure 8 at each redshift (black circles), with the predicted faint-end slope from the same quasar lifetime models (red crosses determined from the observations at each $z$ ). Bottom left shows the total black hole mass density of the Universe, from the data (circles), best fit QLF (solid line). Inset shows the fraction of the $z=0$ mass density $\left(\rho_{\mathrm{BH}}(z) / \rho_{\mathrm{BH}}(0)\right)$, on a linear scale. Green diamond, blue square, and red cross show the local black hole mass density calculated in Yu \& Tremaine (2002); Marconi et al. (2004), and Shankar et al. (2004), respectively. Bottom center shows the integrated black hole mass function at $z=0$ (thick black line) and $z=1,2,3$ (blue, green, and red lines, respectively), compared to the inferred local mass function from Marconi et al. (2004) (yellow shaded range shows $1 \sigma$ range) and Shankar et al. (2004) (thin black line). Bottom right shows the integrated cosmic X-ray background (black line), compared to the observations of Barcons et al. (2000) and Gruber et al. (1999) (blue and red lines, respectively, with shaded yellow $1 \sigma$ observational uncertainty).

then the integrated black hole mass density is given by integrating the luminosity density (Soltan 1982). Figure 10 shows the result of this integration from $z \rightarrow \infty$ to $z$, assuming $\epsilon_{r}=$ 0.1 . We extrapolate beyond $z=6$ but this only changes the $z=$ 0 result by $\approx 0.03$ dex. Note that the vertical axis is logarithmic; on a linear scale most of the growth occurs at $z \lesssim 1-2$, in agreement with previous estimates (e.g., Yu \& Tremaine 2002; Marconi et al. 2004; Shankar et al. 2004).

This gives a $z=0$ black hole mass density of

$$
\rho_{\mathrm{BH}}(z=0)=4.81_{-0.99}^{+1.24}\left(\frac{0.1}{\epsilon_{r}}\right) h_{70}^{2} \times 10^{5} M_{\odot} \mathrm{Mpc}^{-3} \text {. }
$$

Comparing this to calculations from local bulge mass, luminosity, and velocity dispersion functions: $\rho_{\mathrm{BH}}=4.2 \pm 1.1$ (Shankar et al. 2004), $\rho_{\mathrm{BH}}=4.6_{-1}^{+1.9}$ (Marconi et al. 2004), $\rho_{\mathrm{BH}}=2.9 \pm 0.5$ (Yu \& Tremaine 2002) (each in units of $h_{70}^{2} \times 10^{5} M_{\odot} \mathrm{Mpc}^{-1}$ ), the agreement is good. The dominant sources of uncertainty are systematic: Tables 3 and 4 show the integrated $\rho_{\mathrm{BH}}(z=0)$, demonstrating that different fitting functions with similar $\chi^{2} / \nu$ (or e.g., allowing $L_{*}$ to evolve as a polynomial in $z$ instead of $\xi$ ) yields similar factors $\sim 1.5-2$ systematic differences in $\rho_{\mathrm{BH}}(z=0)$. An additional contribution from Compton-thick sources is another uncertainty, although it cannot be much larger or would overpredict the present mass density and X-ray background (below). Fractional uncertainties in $\epsilon_{r}$ are substantial, and e.g. Hopkins. Naravan. \& Hernquist (2006) estimate based on radiatively inefficient accretion flow models (e.g., Naravan \& Yi 1995) a mass-weighted effective $\epsilon_{r}$ a factor of $\sim 0.8$ lower than the "radiatively efficient" value owing to some growth at low Eddington ratio. Given these uncertainties, it is not necessarily meaningful to compare with the local black hole mass density with any greater statistical accuracy.

Having factored out the quasar lifetime in $\S 4.1$ to determine the rate of build-up of individual black holes $\dot{\phi}\left(M_{\mathrm{BH}}\right)$, we can trivially integrate this over redshift to determine the relic black hole mass function (see Hopkins et al. 2006b, for a full derivation of the relevant equations). We show the resulting $z=0$ black hole mass function in Figure 10 compared to the local mass function estimates from Shankar et al. (2004) and Marconi et al. (2004), determined from local bulge velocity dispersion, mass, and luminosity function observations (Marzke et al. 1994; Kochanek et al. 2001; Cole et al. 2001; Nakamura et al. 2003; Blanton et al. 2003; Bernardi et al. 2003; Sheth et al. 2003). The integrated black hole mass function agrees well at all masses. We emphasize that there are no free parameters to fit to the black hole mass function in this analysis, as there are in the traditional analyses of e.g. Yu \& Tremaine (2002). The mass functions at several redshifts are also shown: the highest-mass black holes are formed at redshifts $z \sim 2$, and low mass black holes assemble at $z \leq 1$, in agreement with a number of previous studies (e.g., Merloni 2004; Marconi et al. 2004; Shankar et al. 2004; Hopkins et al. 2006b).

Finally, we can integrate over the bolometric QLF, including our full modeling of spectral shapes and column density distributions to determine the $\mathrm{X}$-ray background spectrum (see also Ueda et al. 2003; Hopkins et al. 2006b). Figure 10 compares this with the observed X-ray background spectrum from Barcons et al. (2000) at $E<10 \mathrm{keV}$ and Gruber et al. (1999) at $E>3 \mathrm{keV}$ (normalized to the same amplitude in the range of overlap based on the detailed compilation in Barcons et al. 2000) with the final $\sim 10 \%$ absolute normalization 
uncertainty shown as the yellow range. The predicted X-ray background spectrum agrees well with that observed over the entire $\sim 1-100 \mathrm{keV}$ range over which "normal" AGN spectra are expected to dominate the X-ray background contribution, and combined with the black hole mass density constraint implies that the X-ray background is dominated by AGN with canonical radiative efficiencies $\epsilon_{r} \sim 0.1$ (compare e.g. Elvis et al. 2002). This also puts strong limits on additional contributions of Compton-thick quasars, as a fraction much larger than the (luminosity-dependent) $\sim 30 \%$ of Ueda et al. (2003) would be problematic.

\subsection{UV and Ionizing Backgrounds}

Figure 11 shows the specific luminosity density $\epsilon_{\nu}$ at $912 \AA$, from our full spectral and obscuration modeling, integrated over our full best-fit QLF model at each redshift. Essentially, this is identical to integrating the $B$-band luminosity functions and using the conversion $L_{\nu}(912 \AA)=$ $10^{18.15} \mathrm{ergs} \mathrm{s}^{-1} \mathrm{~Hz}^{-1}\left(L_{B} / L_{\odot}\right)$, (Elvis et al. 1994; Telfer et al. 2002; Richards et al. 2006c). We also show the bolometric luminosity density from Figure 8 renormalized by a constant "bolometric correction," and the $\epsilon_{\nu}$ implied if this luminosity density were proportional to the number density of bright quasars, $\epsilon_{\nu} \propto \Phi\left(M_{B}<-27\right)$, both normalized to give the inferred $\epsilon_{\nu}$ at $z=3$. The latter correction is given by

$$
\epsilon_{\nu} \approx 0.95 \times 10^{24} \mathrm{ergs} \mathrm{s}^{-1} \mathrm{~Hz}^{-1}\left[\frac{\Phi\left(M_{B}<-27\right)}{10^{-8} \mathrm{Mpc}^{-3}}\right] .
$$

At $z \lesssim 2$, the faint and bright end slopes of the QLF are relatively steep, indicating an important contribution to luminosity densities from faint objects. As a result, $j_{\text {bol }}$ (neglecting obscuration) and $\Phi\left(M_{B}<-27\right)$ (neglecting all faint objects) are poor proxies for $\epsilon_{\nu}$. Above $z \sim 2$, however, the luminosity density is dominated by bright (and relatively unobscured) objects, and Equation (28) provides an accurate approximation to the full calculation of the specific luminosity density from all available constraints in Table 1

Given $\epsilon_{\nu}$ at $912 \AA$ and some description of the IGM opacity, we can estimate the ionization rate contributed by the quasar population. The right panel of Figure 11] shows this rate $\Gamma_{-12}\left(\right.$ rate in $10^{-12}$ ionizations $\mathrm{s}^{-1}$ atom $^{-1}$ ), where we have used the conversions from Fardal et al. (1998); Schirber \& Bullock (2003)

$$
\Gamma_{-12}(z) \approx 2.0(1+z)^{-1.5} \frac{\epsilon_{24}}{3+\left|\alpha_{\mathrm{UV}}\right|}
$$

with $\alpha_{\mathrm{UV}}$ defined in $\S 2.1$ and $\epsilon_{24}=\epsilon_{\nu} /\left(10^{24} \operatorname{ergs~s}^{-1} \mathrm{~Hz}^{-1}\right)$. We compare with several observational estimates of the total $\Gamma_{-12}$ from Scott et al. (2000); McDonald \& Miralda-Escudé (2001, 2003); Tvtler et al. (2004); Bolton et al. (2005); Rollinde et al. (2005); Fan et al. (2005) using various methods (for a review, see Fan et al. 2006). Note that this assumes a specific IGM optical depth, escape fraction, and Lyman Limit System distribution/evolution, which are by no means well-constrained, so we caution that we do not mean this to be a rigorous calculation of $\Gamma_{-12}$ and also show the same estimate multiplied (divided) by a factor of 2 . U1timately, this rough calculation supports previous estimates (e.g., Madau et al. 1999; Fan et al. 2001b; Bolton et al. 2005) that at $z \sim 2$, the ionizing background contributed by quasars is comparable to $(\sim 50 \%)$ that observed, but the quasar contribution drops much more rapidly with redshift than the ionizing background. The contributed $\epsilon_{\nu}$ declines from $z \sim 2-6$ roughly $\propto(1+z)^{-4}$ (giving a factor $\sim 60$ drop in $\Gamma_{-12}$ ), whereas the observed total $\Gamma_{-12}$ falls by only a factor $\sim 10$.

\section{DISCUSSION/CONCLUSIONS}

We have used a large set of observed quasar luminosity functions in various wavebands (Table1), from the IR through optical, soft and hard X-rays, and emission line measurements, combined with recent estimates of the quasar column density distribution from hard X-ray and IR observations, and a large number of observations from the radio through hard Xrays determining the distribution of quasar spectral shapes to estimate the bolometric quasar luminosity function. This allows us to fit the bolometric luminosity function over a wide baseline in both luminosity and redshift, from bolometric luminosities $\sim 10^{41}-10^{49} \mathrm{erg} \mathrm{s}^{-1}$ and redshifts $z=0-6$, reliably determining the location of the break even at $z \sim 3$ and constraining its location with $\gtrsim 2 \sigma$ confidence up to $z \sim 4.5$.

Combining observations at different wavelengths but at the same redshift allows us to test the self-consistency of measurements of the column density distribution and spectral shape. With the best-fit current estimates of the column density distribution and quasar spectral shape, both of which depend on luminosity (the unobscured fraction and optical/UV prominence in the quasar spectrum increasing with luminosity), a single bolometric QLF selfconsistently reproduces the observed QLFs in each available band at all redshifts for which we have compiled measurements. A constant (luminosity-independent) column density distribution cannot self-consistently reproduce the observed QLF in different bands, and is increasingly ruled out by direct observations (Hill. Goodrich, \& DePov 1996; Simpson. Rawlings, \& Lacv 1999; Willott et al.|2000; Simpson \& Rawlings 2000; Steffen et al. 2003; Ueda et al. 2003; Grimes, Rawlings, \& Willott 2004; Hasinger 2004; Sazonov \& Revnivtsev 2004; Barger et al. 2005; Simpson 2005; Hao et al. 2005; Sazonov et al. 2006; Shinozaki et al. 2006; Beckmann et al. 2006b; Bassani et al. 2006). A strong redshift dependence in the column density distribution is also ruled out: measurements of such a dependence (e.g., La Franca et al. 2005) fit the observations with comparable accuracy to a redshift-independent model up to $z \sim$ 1 (where the observations are generally calibrated), but extrapolate poorly to higher redshifts. Likewise, a single quasar spectrum cannot self-consistently reproduce the observed QLFs in different wavebands; it is necessary to account for the dependence of spectral shape on luminosity seen in Wilkes et al. (1994); Green et al. (1995); Vignali et al. (2003); Strateva et al. (2005); Richards et al. (2006c); Steffen et al. (2006).

Extrapolating any fitted luminosity function outside of its measured luminosity and redshift range can be inaccurate by orders of magnitude (see, e.g. Figure 19 of Richards et al. $(2006 \mathrm{~b})$ ), and we have demonstrated the importance of accounting for the detailed luminosity dependence of quasar SEDs and obscuration. It is also important to include the scatter in bolometric corrections, i.e. the fact that there is not one spectral shape even at a given luminosity, as failure to account for this can over-predict the number density of the most luminous quasars by an order of magnitude, and over-predict the bolometric luminosity density by $\sim 30 \%$. Given the large baselines spanned by our compiled samples and our full treatment of these effects, we provide a number of fitting formulae to simplify future comparisons, including: the bolometric QLF itself (Table 3), median bolometric corrections (Equation 2), the dispersion in bolometric corrections (Equation 3), and effective "obscured/visible" fractions (Equation (4) as a 

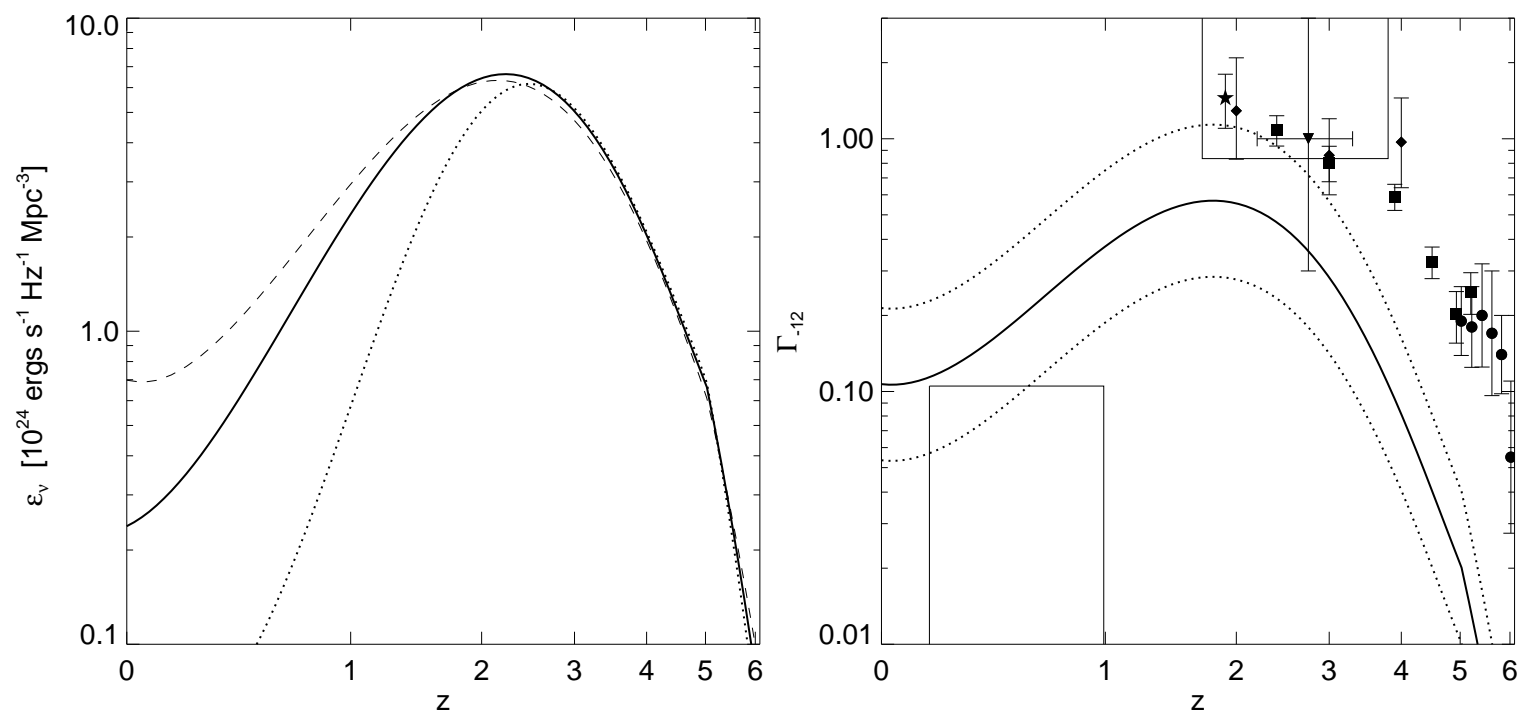

FIG. 11.- Left: Specific luminosity density at $912 \AA$, calculated using our full spectral and obscuration modeling (solid line). If instead we adopt a constant conversion from the bolometric luminosity density in Figure 8 (or, similarly, ignore obscuration) the dashed line is obtained. Taking a constant conversion from the number density of quasars with $M_{B}<-27$ (Equation $[28$ here normalized to give the exact specific luminosity density at $z=3$ ) yields the dotted line. Right: Implied rate of ionization $\Gamma_{-12}$ (in units of $10^{-12} \mathrm{~s}^{-1}$ ) as a function of redshift, from our full modeling (black line), adopting the conversions from $\epsilon_{\nu}$ from Fardal et al. (1998); Schirber \& Bullock (2003). Dotted lines show the same, but arbitrarily increased (decreased) by a factor of 2 . Observational estimates of $\Gamma_{-12}$ are shown, from Bolton et al. (2005) (diamonds at $z=2-4$ ), Tvtler et al. (2004) (star at $z=1.9$ ), Rollinde et al. (2005) (triangle at $z=2.75$ ), McDonald \& Miralda-Escudé 2001, 2003) (squares at $z=2.4-5.2$ ), Fan et al. (2005) (circles at $z=5-6$ ), and Scott et al. (2000) (boxes at $z \sim 0-1$ and $z \sim 2-4$ ). Below $z \sim 2$, the faint quasar contribution is important, with the effects of obscuration and a changing faint end slope requiring a detailed calculation of the specific ionizing flux. However, above $z \sim 2$, the flatter bright and faint end slopes imply that bright quasars dominate the luminosity density, and the evolution in the number density of bright quasars becomes an accurate proxy for the evolution in $\epsilon_{\nu}$. Quasars can account for all of the observationally estimated $\Gamma_{-12}$ at $z \lesssim 1$, and $\sim 50 \%$ at $z \sim 2-3$ (for the specific conversions adopted), but the quasar luminosity density drops off much faster than $\Gamma_{-12}$ at higher $z$.

function of luminosity.

We find that the faint-end slope of the QLF flattens at increasing redshift, at high significance $(>7 \sigma)$. Evidence for such a trend comes from a number of different measurements (Page et al.|1997; Mivaii et al. 2000, 2001; La Franca et al. 2002; Cowie et al. 2003; Ueda et al. 2003; Fiore et al. 2003; Hunt et al. 2004; Cirasuolo et al. 2005; Hasinger et al. 2005) (although we still find $\sim 3 \sigma$ evidence for this evolution without these data), but independently these samples detect such evolution with only marginal $(\lesssim 2 \sigma)$ significance. The most significant detection from a single study thus far has been that of Hasinger et al. (2005), who find an improvement from a $\sim 5 \%$ 2D K-S probability to $\sim 36 \%$ allowing for faint-end evolution; it is simply difficult to obtain the large baseline necessary for strong constraints from a single sample. There has also been some debate over whether the steep faint-end slopes seen at $z \lesssim 0.2$ by e.g. Sazonov \& Revnivtsev (2004); Hao et al. (2005) are consistent with those seen in other (particularly optical) surveys, and indeed Figure 6 demonstrates they are not consistent with the PLE model. However, accounting for the evolution in the faint end slope with redshift, Figure 6 demonstrates that these observations are consistent with the others we compile. Our analysis also allows us to understand the different shapes of the QLF in different observed bands, and Figures 4,6 \& 7 demonstrate that with obscuration more important in low-luminosity quasars, the faint-end optical QLF is relatively flattened at all redshifts. Together with optical samples generally not probing to luminosities as faint as the X-ray samples, this explains why the trend of evolution in the faint-end slope has been preferentially observed in X-ray samples.

This confirms, at high significance, that there is faint-end evolution in the data. This does not, of course, imply that the evolution is necessarily real, because selection effects such as incompleteness at low luminosities and high redshifts, or an evolving Compton-thick population, could play a role. However, constraints on this quantity are interesting. For example, to the extent that the evolution is real, it supports at much greater significance the findings of Hopkins et al. (2006a), namely the predicted functional dependence of the faint-end shape on $L_{*}$ (and, as a consequence, on redshift). This model is motivated by hydrodynamic simulations (Hopkins et al. $2005 a, b, c)$ and analytical models of accretion rate evolution in feedback-driven outflows (Hopkins \& Hernquist 2006), which predict both that the faint-end shape should be controlled by the shape of the quasar light curve, itself a function of quasar and host galaxy properties. To the extent that the faint-end slope does or does not evolve, it directly constrains the quasar "lifetime" as a function of luminosity (i.e. differential time quasars spend per luminosity interval in both growth and decay; Hopkins et al. 2005c), and consequently feedback coupling modes and their impact on the host galaxy.

We also find at high significance $(>6 \sigma)$ that the bright end slope of the QLF evolves with redshift, becoming shallower at high redshifts $z \geq 3$. This agrees with the early suggestions of Schmidt et al. (1995); Fan et al. (2001a b), and subsequent more robust measurements of Richards et al. (2006b). We infer significant $(>3 \sigma)$ evidence for such evolution even with those samples removed $\left(k_{\gamma_{1}, 1}=1.22 \pm 0.15, k_{\gamma_{1}, 2}=-0.80 \pm\right.$ 0.09 ), but in this case the result is heavily influenced by the $\mathrm{X}$-ray data, which at the highest luminosities has a large bolometric correction $\left(L_{\mathrm{bol}} \sim 100 L_{H X}\right.$, from the observed $\alpha_{\mathrm{ox}}-L$ relation). Since the QLF is falling steeply at these luminosities, a small change in the bolometric correction will signifi- 
cantly change the inferred slope; Figure 4 aptly demonstrates that the optical and IR samples provide a much more robust probe of the "true" bright end slope. Note that the best-fit trend at $z \sim 6$ favors a shallower slope than recently suggested by Fan et al. (2004); Cool et al. (2006), although the small number of objects make this only a $\sim 1 \sigma$ effect. Further wide-area surveys are needed to confirm the trend.

There is also marginal evidence that the bright-end QLF slope is somewhat shallow at $z \sim 0$, steepening to $z \sim 0.5-1$, then becoming shallower with $z$ as described above. This low-redshift trend is marginally evident in the Richards et al. (2006b) sample as well (see Figure 8), although with a smaller low $-z$ baseline the authors assumed a constant bright-end slope up to $z \sim 3$. There are a number of potential systematic effects which complicate understanding this trend. For example, imperfect accounting of the (potentially quite rapid) QLF evolution over a narrow redshift interval will bias the luminosity function to a higher number density at large $L$, as will many general binning procedures used in presenting the data, as well as host galaxy contamination (see e.g. Figure 11 of Hao et al. 2005). These problems are well known from local galaxy luminosity functions, and can introduce significant effects even in samples spanning the range e.g. $z \sim 0-0.1$ (see e.g.,Blanton et al.2003), where many of the low-redshift QLF measurements cover a significantly larger $z \sim 0-0.3$. Furthermore, the faint-end slope is observed to be steep at $z \sim 0$ (e.g., Hao et al. 2005), implying a weak break and large covariance between the best-fit parameters (much larger than at any other redshifts where the break is constrained). More careful re-analysis of these samples and e.g. recent local luminosity functions from Shinozaki et al. (2006), Sazonov et al. (2006), and Beckmann et al. (2006b), accounting for these effects, is needed.

Again, despite systematic uncertainties, evolution in the bright-end shape is interesting. Models predict that it should evolve with redshift, as a consequence of feedback from AGN (e.g., Kauffmann \& Haehnelt 2000; Wvithe \& Loeb 2003; Scannapieco et al. 2005). At high redshift, with small galaxies, large gas content, and rapid merger rates, the quasar activity is expected to trace dark matter halo growth and merger rates, until a redshift $z \sim 2-3$ when feedback from quasar growth or other mechanisms begins to dominate. This "shuts down" activity, terminates subsequent black hole growth and local star formation (Hopkins et al. 2006c; Springel et al. 2005a), injects entropy (Scannapieco \& Oh 2004) and radiomode feedback from low accretion rate "dying" black holes (Croton et al. 2006) heats the surrounding IGM and prevents new infall, suppressing further galaxy and $\mathrm{BH}$ growth. In these models, this occurs first in the largest systems, which evolve the most rapidly and have the most massive, violent black holes, leaving a gas-rich merger and corresponding quasar history which shifts to lower masses at lower redshifts (Hopkins et al. 2006d), and building up lower-mass elliptical galaxies at preferentially later times (Hopkins et al. 2006c d e), explaining observed trends of "cosmic downsizing" (e.g., Cowie et al. 1996) in the context of hierarchical structure formation.

Our estimate of the bolometric QLF allows us to immediately derive several interesting quantities. The bolometric quasar luminosity density (and correspondingly, total black hole accretion rate) of the Universe follows a similar qualitative trend to that of $L_{*}$, peaking at a well-defined $z \approx 2.154 \pm$ 0.052. The integrated black hole mass density to $z=0$ is consistent with local estimates from bulge mass and velocity dispersion functions (e.g., Marconi et al. 2004; Shankar et al. 2004), with room for observationally estimated Comptonthick fractions of $\sim 30-40 \%$ which we have included (but not, e.g. $\gtrsim 60 \%$ ). The predicted X-ray background spectrum agrees well with that observed, in a similar manner to that in Ueda et al. (2003). The UV luminosity density and ionization rate are consistent with all existing constraints, and account for observed ionization rates from Scott et al. (2000) at $z \lesssim 1$ and a significant $(\sim 50 \%)$ fraction at $z \sim 2-3$, but subsequently dropping more rapidly than observed rates. Because of the flattening of the bright and faint-end QLF slopes with redshift, the luminosity density at high redshift is dominated by bright quasars, and above $z \sim 2.5$ the number density of bright, optical quasars (e.g. $M_{B}<-27$ ) becomes an accurate proxy for the UV luminosity density, related by a conversion which we calibrate (see $\S 4.3$ ).

We can also de-convolve the bolometric QLF with the quasar lifetime as a function of luminosity and black hole mass; similar to the procedure in e.g. Yu \& Tremaine (2002) but adopting the physically motivated quasar lifetimes from Hopkins et al. (2006a b) to determine the "birthrate" (formation rate) of black holes as a function of mass and redshift. The resulting evolution of the black hole mass function illustrates the feedback-driven scenario above, with rapid buildup to $z \sim 3$, then activity shutting down in the highest mass systems, and the characteristic mass of black holes "in formation" shifting from $\sim 10^{9} M_{\odot}$ at $z \sim 2$ to $\sim 2 \times 10^{7} M_{\odot}$ at $z \sim 0$. The resulting black hole mass function agrees well with that inferred locally (Marconi et al. 2004; Shankar et al. 2004).

Measurements of the QLF are converging towards a selfconsistent picture of the QLF shape and evolution across $\sim 8$ orders of magnitude in luminosity and $\sim 9$ in space density, frequencies from $\sim 24 \mu \mathrm{m}$ to $\sim 100 \mathrm{keV}$, through mid and near-IR, optical, UV, soft and hard X-rays. Except at the highest redshifts $z \gtrsim 4$, where statistics are still poor and faint luminosities have not been probed, systematic errors will almost certainly overwhelm statistical errors. Indeed, comparison of samples with overlapping redshift and luminosity intervals shows that while the agreement is good, the systematic offsets (albeit small) between the largest samples are much larger than their typical statistical errors. Some of these effects, such as cosmic variance, are at least straightforward to understand and reduce. However, others are more fundamental. It may not be possible to define a bolometric QLF to any greater accuracy than we have done (again, excepting the highest redshifts where larger samples would improve things considerably), at least in the near future.

For example, on both small scales characteristic of accretion disks, black hole driven winds, and molecular outflows or tori, and large scales characteristic of potential mergerdriving and galaxy-scale quasar obscuration, quasars are not isotropic. So even a complete spectrum does not imply the bolometric luminosity, lacking a more complete model for the spectrum as a function of viewing angle and other quantities (e.g. accretion rate, mass, spin). Perhaps by considering the distribution of luminosities and accounting for the fact that there is not one spectral shape for a given total bolometric luminosity, we have statistically accounted for some of this, but it is by no means clear, and will not necessarily be so if there are correlations between e.g. the column density distribution and intrinsic Eddington ratio or spectral shape distribution, or if e.g. the dependence of spectral shape on luminosity is really driven by an accretion rate or viewing angle dependence. 
These are important but difficult and often degenerate questions, which can only be answered with more detailed observations of quasar spectra at many different frequencies, coupled with detailed modeling of accretion processes and quasar fueling.

Finally, in order to enable simple comparison of our results with future theoretical models and observations at a wide variety of wavelengths and redshifts, we provide for public use ${ }^{4}$ a simple "QLF calculator" script to return the QLF at a given redshift, in an arbitrary observed band or frequency. This calculates the observed QLF using the full modeling of bolometric corrections and extinction discussed herein, for any of the QLF fits in Tables 3 \& 4 or for an arbitrary bolometric QLF. As we have emphasized for the large number of historical fits to the QLF, the fits therein should not be extrapolated beyond the observed ranged in Figures $6 \& \square$ for example to very high redshifts $(z \gtrsim 6)$, low luminosities at high redshifts $(z \gtrsim 4.5)$, and unconstrained wavelength regimes such as the extreme IR, UV, or hard X-rays.

We thank Günther Hasinger for helpful discussions, as well as T. J. Cox, Brant Robertson, and Scott Tremaine. We are also grateful to an anonymous referee for comments that improved this paper. This work was supported in part by NSF grants ACI 96-19019, AST 00-71019, AST 02-06299, and AST 03-07690, and NASA ATP grants NAG5-12140, NAG513292, and NAG5-13381. GTR further acknowledges support from a Gordon and Betty Moore Fellowship in data intensive sciences.

\footnotetext{
${ }^{4}$ http://www. cfa.harvard.edu/ phopkins/Site/qlf.html
}

\section{REFERENCES}

Ballantyne, D. R., Everett, J. E., \& Murray, N. 2006, ApJ, 639, 740 Barcons, X., Mateos, S., \& Ceballos, M. T. 2000, MNRAS, 316, L13 Barger, A. J., et al. 2003a, AJ, 126, 632

Barger, A. J., Cowie, L. L., Capak, P., Alexander, D. M., Bauer, F. E., Brandt, W. N., Garmire, G. P., \& Hornschemeier, A. E. 2003b, ApJ, 584, L61 Barger, A. J., \& Cowie, L. L. 2005, ApJ, 635, 115

Barger, A. J., Cowie, L. L., Mushotzky, R. F., Yang, Y., Wang, W.-H., Steffen, A. T., \& Capak, P. 2005, AJ, 129, 578

Bassani, L., et al. 2006, ApJ, 636, L65

Bechtold, J., et al. 2003, ApJ, 588, 119

Beckmann, V., Gehrels, N., Shrader, C. R., \& Soldi, S. 2006a, ApJ, 638, 642 Beckmann, V., Soldi, S., Shrader, C. R., Gehrels, N., \& Produit, N. 2006b, $\mathrm{ApJ}$, in press astro-ph/0606687

Bernardi, M., et al. 2003, AJ, 125, 1849

Blanton, M. R., et al. 2003, ApJ, 592, 819

Bolton, J. S., Haehnelt, M. G., Viel, M., \& Springel, V. 2005, MNRAS, 357, 1178

Boyle, B. J., Shanks, T., \& Peterson, B. A. 1988, MNRAS, 235, 935

Boyle, B. J., Shanks, T., Croom, S. M., Smith, R. J., Miller, L., Loaring, N., \& Heymans, C. 2000, MNRAS, 317, 1014

Brotherton, M. S., Tran, H. D., Becker, R. H., Gregg, M. D., LaurentMuehleissen, S. A., \& White, R. L. 2001, ApJ, 546, 775

Brown, M. J. I., et al. 2006, ApJ, 638, 88

Cirasuolo, M., Magliocchetti, M., \& Celotti, A. 2005, MNRAS, 357, 1267

Cole, S., et al. 2001, MNRAS, 326, 255

Comastri, A., Setti, G., Zamorani, G., \& Hasinger, G. 1995, A\&A, 296, 1

Comerford, J. M., Haiman, Z., \& Schaye, J. 2002, ApJ, 580, 63

Cool, R. J., et al. 2006, AJ, 132, 823

Cowie, L. L., Songaila, A., Hu, E. M., \& Cohen, J. G. 1996, AJ, 112, 839

Cowie, L. L., Barger, A. J., Bautz, M. W., Brandt, W. N., \& Garmire, G. P. 2003, ApJ, 584, L57

Cristiani, S., et al. 2004, ApJ, 600, L119

Croom, S. M., Smith, R. J., Boyle, B. J., Shanks, T., Miller, L., Outram, P. J., \& Loaring, N. S. 2004, MNRAS, 349, 1397

Croton, D. J., et al. 2006, MNRAS, 365, 11

Di Matteo, T., Springel, V., \& Hernquist, L. 2005, Nature, 433, 604

Ellison, S. L., Hall, P. B., \& Lira, P. 2005, AJ, 130, 1345

Elvis, M., et al. 1994, ApJS, 95, 1

Elvis, M., Risaliti, G., \& Zamorani, G. 2002, ApJ, 565, L75

Fan, X., et al. 2001a, AJ, 121, 54

Fan, X., et al. 2001b, AJ, 122, 2833

Fan, X., et al. 2003, AJ, 125, 1649

Fan, X., et al. 2004, AJ, 128, 515

Fan, X., et al. 2005, AJ, in press astro-ph/0512082

Fan, X., Carilli, C. L., \& Keating, B. 2006, ARA\&A, in press astro-ph/0602375

Fardal, M. A., Giroux, M. L., \& Shull, J. M. 1998, AJ, 115, 2206

Ferrarese, L. \& Merritt, D. 2000, ApJ, 539, L9

Fioc, M. \& Rocca-Volmerange, B. 1997, A\&A, 326, 950

Fiore, F., et al. 2003, A\&A, 409, 79

Gebhardt, K., Bender, R., Bower, G. et al. 2000, ApJ, 539, L13

George, I. M., Turner, T. J., Netzer, H., Nandra, K., Mushotzky, R. F., \& Yaqoob, T. 1998, ApJS, 114, 73

Gilli, R., Risaliti, G., \& Salvati, M. 1999, A\&A, 347, 424
Granato, G. L., De Zotti, G., Silva, L., Bressan, A., \& Danese, L. 2004, ApJ, 600,580

Green, P. J., et al. 1995, ApJ, 450, 51

Gregg, M. D., Lacy, M., White, R. L., Glikman, E., Helfand, D., Becker, R. H., \& Brotherton, M. S. 2002, ApJ, 564, 133

Grimes, J. A., Rawlings, S., \& Willott, C. J. 2004, MNRAS, 349, 503

Gruber, D. E., Matteson, J. L., Peterson, L. E., \& Jung, G. V. 1999, ApJ, 520, 124

Haas, M., et al. 2004, A\&A, 424, 531

Hao, L., et al. 2005, AJ, 129, 1795

Hartwick, F. D. A., \& Schade, D. 1990, ARA\&A, 28, 437

Hasinger, G. 2004, Nucl. Phys. B Proc. Supp., 132, 86

Hasinger, G., Miyaji, T., \& Schmidt, M. 2005, A\&A, 441, 417

Hatziminaoglou, E., et al. 2005, AJ, 129, 1198

Heckman, T. M., Ptak, A., Hornschemeier, A., \& Kauffmann, G. 2005, ApJ, 634,161

Hewett, P. C., Foltz, C. B., \& Chaffee, F. H. 1993, ApJ, 406, L43

Hill, G. J., Goodrich, R. W., \& DePoy, D. L. 1996, ApJ, 462, 163

Hopkins, P. F., et al. 2004, AJ, 128, 1112

Hopkins, P. F., Hernquist, L., Martini, P., Cox, T. J., Robertson, B., Di Matteo, T., \& Springel, V. 2005a, ApJ, 625, L71

Hopkins, P. F., Hernquist, L., Cox, T. J., Robertson, B., Di Matteo, T., Martini, P., \& Springel, V. 2005b, ApJ, 630, 705

Hopkins, P. F., Hernquist, L., Cox, T. J., Robertson, B., Di Matteo, T., \& Springel, V. 2005c, ApJ, 630, 716

Hopkins, P. F., Hernquist, L., Cox, T. J., Robertson, B., Di Matteo, T., \& Springel, V. 2005d, ApJ, 632, 81

Hopkins, P. F., Hernquist, L., Cox, T. J., Robertson, B., Di Matteo, T., \& Springel, V. 2006a, ApJ, 639, 700

Hopkins, P. F., Hernquist, L., Cox, T. J., Robertson, B., Di Matteo, T., \& Springel, V. 2006b, ApJS, 163, 1

Hopkins, P. F., Hernquist, L., Cox, T. J., Robertson, B., \& Springel, V. 2006c, ApJS, 163, 50

Hopkins, P. F., Bundy, K., Hernquist, L., Ellis, R. S., 2006d, ApJ, submitted, astro-ph/0601621

Hopkins, P. F., Somerville, R. S., Hernquist, L., Cox, T. J., Robertson, B., \& Li, Y. 2006e, ApJ, submitted astro-ph/0602290

Hopkins, P. F., \& Hernquist, L. 2006, ApJS, submitted astro-ph/0603180

Hopkins, P. F., Narayan, R., Hernquist, L. 2006, ApJ, accepted astro-ph/0510369

Hunt, M. P., Steidel, C. C., Adelberger, K. L., \& Shapley, A. E. 2004, ApJ, 605,625

Jiang, L., et al. 2006a, AJ, 131, 2788

Jiang, L., et al. 2006b, AJ, in press |astro-ph/0608006

Kauffmann, G., \& Haehnelt, M. 2000, MNRAS, 311, 576

Kennefick, J. D., Djorgovski, S. G., \& de Carvalho, R. R. 1995, AJ, 110, 2553

Kochanek, C. S., et al. 2001, ApJ, 560, 566

Koo, D. C., \& Kron, R. G. 1988, ApJ, 325, 92

Kormendy, J. \& Richstone, D. 1995, ARA\&A, 33, 581

Kuhn, O., Elvis, M., Bechtold, J., \& Elston, R. 2001, ApJS, 136, 225

La Franca, F., et al. 2002, ApJ, 570, 100

La Franca, F., et al. 2005, ApJ, 635, 864

Lawrence, A. 1991, MNRAS, 252, 586

Madau, P., Haardt, F., \& Rees, M. J. 1999, ApJ, 514, 648 
Magdziarz, P., \& Zdziarski, A. A. 1995, MNRAS, 273, 837

Magorrian, J. et al. 1998, AJ, 115, 2285

Mainieri, V., et al. 2005, A\&A, 437, 805

Maiolino, R., Schneider, R., Oliva, E., Bianchi, S., Ferrara, A., Mannucci, F., Pedani, M., \& Roca Sogorb, M. 2004, Nature, 431, 533

Marconi, A., \& Hunt, L. K. 2003, ApJ, 589, L21

Marconi, A., Risaliti, G., Gilli, R., Hunt, L. K., Maiolino, R., \& Salvati, M. 2004, MNRAS, 351, 169

Markwardt, C. B., Tueller, J., Skinner, G. K., Gehrels, N., Barthelmy, S. D., \& Mushotzky, R. F. 2005, ApJ, 633, L77

Marzke, R. O., Geller, M. J., Huchra, J. P., \& Corwin, H. G. 1994, AJ, 108, 437

Matute, I., La Franca, F., Pozzi, F., Gruppioni, C., Lari, C., \& Zamorani, G. 2006, A\&A, in press astro-ph/0601355

McDonald, P., \& Miralda-Escudé, J. 2001, ApJ, 549, L11

McDonald, P., \& Miralda-Escudé, J. 2003, ApJ, 595, L67

McLure, R. J. \& Dunlop, J. S. 2004, MNRAS, 352, 1390

Merloni, A. 2004, MNRAS, 353, 1035

Miyaji, T., Hasinger, G., \& Schmidt, M. 2000, A\&A, 353, 25

Miyaji, T., Hasinger, G., \& Schmidt, M. 2001, A\&A, 369, 49

Morrison, R. \& McCammon, D. 1983, ApJ, 270, 119

Nakamura, O., Fukugita, M., Yasuda, N., Loveday, J., Brinkmann, J., Schneider, D. P., Shimasaku, K., \& SubbaRao, M. 2003, AJ, 125, 1682

Nandra, K., Laird, E. S., \& Steidel, C. C. 2005, MNRAS, 360, L39

Narayan, R. \& Yi, I. 1995, ApJ, 452, 710

Page, M. J., Mason, K. O., McHardy, I. M., Jones, L. R., \& Carrera, F. J. 1997, MNRAS, 291, 324

Pei, Y. C. 1992, ApJ, 395, 130

Pei, Y. C. 1995, ApJ, 438, 623

Peng, C. Y., et al. 2006, ApJ astro-ph/0603248

Perola, G. C., Matt, G., Cappi, M., Fiore, F., Guainazzi, M., Maraschi, L., Petrucci, P. O., \& Piro, L. 2002, A\&A, 389, 802

Peterson, B. M. 1997, An introduction to active galactic nuclei, Publisher: Cambridge, New York Cambridge University Press, 1997 Physical description xvi, 238

Richards, G. T., Fan, X., Schneider, D. P., Vanden Berk, D. E., Strauss, M. A., York, D. G., Anderson, J. E., Anderson, S. F., et al. 2001, AJ, 121, 2308

Richards, G. T., et al. 2003, AJ, 126, 1131

Richards, G. T., et al. 2005, MNRAS, 360, 839

Richards, G. T., et al. 2006a, AJ, 131, 49

Richards, G. T., et al. 2006b, AJ, 131, 2766

Richards, G. T., et al. 2006c, ApJS, in press astro-ph/0601558

Risaliti, G., \& Elvis, M. 2005, ApJ, 629, L17

Risaliti, G., Maiolino, R., \& Salvati, M. 1999, ApJ, 522, 157

Rollinde, E., Srianand, R., Theuns, T., Petitjean, P., \& Chand, H. 2005, MNRAS, 361, 1015

Salucci, P., Szuszkiewicz, E., Monaco, P., \& Danese, L. 1999, MNRAS, 307, 637

Sazonov, S., Revnivtsev, M., Krivonos, R., Churazov, E., \& Sunyaev, R. 2006, A\&A, in press astro-ph/0608418

Sazonov, S. Y., \& Revnivtsev, M. G. 2004, A\&A, 423, 469

Scannapieco, E., \& Oh, S. P. 2004, ApJ, 608, 62

Scannapieco, E., Silk, J., \& Bouwens, R. 2005, ApJ, 635, L13

Schirber, M., \& Bullock, J. S. 2003, ApJ, 584, 110

Schmidt, M. 1968, ApJ, 151, 393

Schmidt, M., \& Green, R. F. 1983, ApJ, 269, 352

Schmidt, M., Schneider, D. P., \& Gunn, J. E. 1995, AJ, 110, 68
Scott, J., Bechtold, J., Dobrzycki, A., \& Kulkarni, V. P. 2000, ApJS, 130, 67 Shankar, F., Salucci, P., Granato, G. L., De Zotti, G., \& Danese, L. 2004 MNRAS, 354, 1020

Shemmer, O., et al. 2006, ApJ, in press astro-ph/0602442

Sheth, R. K., et al. 2003, ApJ, 594, 225

Shinozaki, K., Miyaji, T., Ishisaki, Y., Ueda, Y., \& Ogasaka, Y. 2006, AJ, 131, 2843

Siana, B. et al. 2006, to appear in Proc. "Spitzer Science Center 2005 Conference: Infrared Diagnostics of Galaxy Evolution,” Pasadena, Ed. R. Chary, in press astro-ph/0604373

Silk, J. \& Rees, M. J. 1998, A\&A, 331, L1

Silverman, J. D., et al. 2005a, ApJ, 618, 123

Silverman, J. D., et al. 2005b, ApJ, 624, 630

Silverman, J., et al. 2005c, to appear in Proc. "The X-ray Universe 2005,"

San Lorenzo de El Escorial, Spain, in press astro-ph/0511552

Simpson, C. 2005, MNRAS, 360, 565

Simpson, C., \& Rawlings, S. 2000, MNRAS, 317, 1023

Simpson, C., Rawlings, S., \& Lacy, M. 1999, MNRAS, 306, 828

Soltan, A. 1982, MNRAS, 200, 115

Spergel, D. N., et al. 2003, ApJS, 148, 175

Spergel, D. N., et al. 2006, ApJ, in press astro-ph/0603449

Springel, V., Di Matteo, T., \& Hernquist, L. 2005a, ApJ, 620, L79

Springel, V., Di Matteo, T., \& Hernquist, L. 2005b, MNRAS, 361, 776

Steffen, A. T., Barger, A. J., Cowie, L. L., Mushotzky, R. F., \& Yang, Y. 2003, ApJ, 596, L23

Steffen, A. T., Strateva, I., Brandt, W. N., Alexander, D. M., Koekemoer, A. M., Lehmer, B. D., Schneider, D. P., \& Vignali, C. 2006, AJ, 131, 2826 Strateva, I. V., Brandt, W. N., Schneider, D. P., Vanden Berk, D. G., \& Vignali, C. 2005, AJ, 130, 387

Telfer, R. C., Zheng, W., Kriss, G. A., \& Davidsen, A. F. 2002, ApJ, 565, 773

Tozzi, P., et al. 2006, A\&A, in press astro-ph/0602127

Treister, E., et al. 2004, ApJ, 616, 123

Treister, E., \& Urry, C. M. 2005, ApJ, 630, 115

Tytler, D., et al. 2004, ApJ, 617, 1

Ueda, Y., Akiyama, M., Ohta, K., \& Miyaji, T. 2003, ApJ, 598, 886

Vanden Berk, D. E., et al. 2001, AJ, 122, 549

Vanden Berk, D. E., et al. 2006, AJ, 131, 84

Vignali, C., Brandt, W. N., \& Schneider, D. P. 2003, AJ, 125, 433

Wang, J. X., \& Jiang, P. 2006, ApJ, 646, L103

Warren, S. J., Hewett, P. C., \& Osmer, P. S. 1994, ApJ, 421, 412

Webster, R. L., Francis, P. J., Peterson, B. A., Drinkwater, M. J., \& Masci, F. J. 1995, Nature, 375, 469

Wilkes, B. J., Tananbaum, H., Worrall, D. M., Avni, Y., Oey, M. S., \& Flanagan, J. 1994, ApJS, 92, 53

Willott, C. J., Rawlings, S., Blundell, K. M., Lacy, M. 2000, MNRAS, 316, 449

Wolf, C., Wisotzki, L., Borch, A., Dye, S., Kleinheinrich, M., \& Meisenheimer, K. 2003, A\&A, 408, 499

Wyithe, J. S. B. 2004, MNRAS, 351, 1266

Wyithe, J. S. B., \& Loeb, A. 2002, ApJ, 577, 57

Wyithe, J. S. B., \& Loeb, A. 2003, ApJ, 595, 614

York, D. G., et al. 2000, AJ, 120, 1579

Yu, Q., \& Lu, Y. 2004, ApJ, 602, 603

Yu, Q., \& Tremaine, S. 2002, MNRAS, 335, 965 
TABLE 1

MEASUREMENTS OF THE QLF

\begin{tabular}{|c|c|c|c|c|c|c|c|}
\hline Reference & Survey/Field ${ }^{\mathrm{a}}$ & Rest Wavelength/Band & $z$ Range $^{\mathrm{b}}$ & Luminosity Range $^{\mathrm{b}}$ & $\chi^{2} / \nu^{\mathrm{c}}$ & $N_{\mathrm{AGN}}$ & Plotting Symbol \\
\hline \multicolumn{8}{|l|}{ Optical: } \\
\hline $\begin{array}{l}\text { Cristiani et al. (2004) } \\
\text { Croom et al. (2004) } \\
\text { Fan et al. (2001a) } \\
\text { Fan et al. (2001b, 2003, 2004) } \\
\text { Hunt et al. (2004) } \\
\text { Kennefick et al. (1995) } \\
\text { Richards et al. (2005) } \\
\text { Richards et al. (2006b) } \\
\text { Schmidt et al. (1995) } \\
\text { Siana et al. (2006) } \\
\text { Wolf et al. (2003) }\end{array}$ & $\begin{array}{l}\text { GOODS } \\
\text { 2QZ/6QZ } \\
\text { SDSS (Equatorial Stripe) } \\
\text { SDSS (Main \& Southern Survey) } \\
\text { LBG survey } \\
\text { POSS } \\
\text { 2dF-SDSS } \\
\text { SDSS (DR3) } \\
\text { PTGS } \\
\text { SWIRE (ELIAS-N1/N2) } \\
\text { COMBO-17 }\end{array}$ & $\begin{array}{c}1450 \AA \\
B \\
1450 \AA \\
1450 \AA \\
1450 \AA \\
B \\
g \\
i(z=2) \stackrel{\sim}{\sim} 2500 \AA \\
B \\
1450 \AA \\
1450 \AA\end{array}$ & $\begin{array}{l}\sim 4-5.2 \\
0.4-2.1 \\
3.6-5.0 \\
\sim 5.7-6.4 \\
\sim 2-4 \\
4.0-4.5 \\
0.3-2.2 \\
0.3-5.0 \\
\sim 3.5-4.5 \\
\sim 2.8-3.4 \\
1.2-4.8\end{array}$ & $\begin{array}{c}-21>M_{1450}>-23.5 \\
-20.5>M_{g}>-28.5 \\
-25.5>M_{1450}>-27.5 \\
-26.5>M_{1450}>-28 \\
-21>M_{1450}>-27 \\
-26.5>M_{B}>-28.5 \\
-21>M_{g}>-27 \\
-22.5>M_{i}>-29 \\
-25.5>M_{B}>-27.5 \\
-23.5>M_{1450}>-26.5 \\
-23.5>M_{1450}>-28.5\end{array}$ & $\begin{array}{c}0.58 / 1 \\
23.1 / 10 \\
6.21 / 9 \\
2.12 / 3 \\
4.74 / 6 \\
14.8 / 2 \\
137 / 99 \\
247 / 101 \\
8.04 / 4 \\
4.74 / 6 \\
54.2 / 27\end{array}$ & $\begin{array}{c}1-4 \\
20,905 \\
39 \\
9 \\
11 \\
10 \\
5,645 \\
15,343 \\
8 \\
\sim 100 \\
192\end{array}$ & $\begin{array}{c}\text { crosses } \\
\text { asterisks } \\
\text { pentagons } \\
\ldots \\
\text { diamonds } \\
\text { triangles } \\
\text { circles } \\
\text { squares } \\
\text { inverted triangles } \\
\text { crosses } \\
\text { stars }\end{array}$ \\
\hline \multicolumn{8}{|l|}{ Soft X-ray: } \\
\hline $\begin{array}{l}\text { Hasinger et al. }(2005) \\
\text { Miyaji et al. }(2000,2001) \\
\text { Silverman et al. }(2005 b) \\
\text { Hard X-ray: }\end{array}$ & $\begin{array}{l}\text { ROSAT }(\mathrm{RASS}+\mathrm{RDS})+\mathrm{CDF}-\mathrm{N} / \mathrm{S} \\
\text { ROSAT }(\mathrm{RASS}+\mathrm{RDS}) \\
\mathrm{CHAMP}+\text { ROSAT }(\mathrm{RASS})\end{array}$ & $\begin{array}{l}0.5-2 \mathrm{keV} \\
0.5-2 \mathrm{keV} \\
0.5-2 \mathrm{keV}\end{array}$ & $\begin{array}{l}0.015-4.8 \\
0.015-4.8 \\
0.1-5\end{array}$ & $\begin{array}{c}10^{42}<L_{0.5-2}<10^{48} \mathrm{erg} \mathrm{s}^{-1} \\
10^{41}<L_{0.5-2}<10^{47} \mathrm{erg} \mathrm{s}^{-1} \\
10^{44.5}<L_{0.5-2}<10^{46} \mathrm{erg} \mathrm{s}^{-1}\end{array}$ & $\begin{array}{l}169 / 51 \\
112 / 41 \\
24.1 / 9\end{array}$ & $\begin{array}{c}2,566 \\
691 \\
217\end{array}$ & $\begin{array}{l}\text { circles } \\
\text { stars } \\
\text { squares }\end{array}$ \\
\hline $\begin{array}{l}\text { Barger et al. (2003a,b) } \\
\text { Barger et al. (2005) } \\
\ldots \\
\text { Barger \& Cowie (2005) } \\
\text { La Franca et al. (2005) } \\
\text { Nandra et al. (2005) } \\
\text { Sazonov \& Revnivtsev (2004) } \\
\text { Silverman et al. (2005a,c) } \\
\text { Ueda et al. (2003) }\end{array}$ & $\begin{array}{l}\text { CDF-N } \\
\text { CDF-N/S + CLASXS + ASCA } \\
\text { CDF-N/S + CLASXS } \\
\text { CDF-N/GOODS-N } \\
\text { HELLAS2XMM } \\
\text { GWS + HDF-N } \\
\text { RXTE } \\
\text { CHAMP } \\
\quad H E A O 1+\text { AMSS-n/s + ALSS } \\
\quad+A S C A+\text { CDF-N }\end{array}$ & $\begin{array}{c}2-8 \mathrm{keV} \\
2-8 \mathrm{keV} \\
2-8 \mathrm{keV} \\
2-8 \mathrm{keV} \\
2-10 \mathrm{keV} \\
2-10 \mathrm{keV} \\
3-20 \mathrm{keV} \\
0.3-8.0 \mathrm{keV} \\
2-10 \mathrm{keV}\end{array}$ & $\begin{array}{l}\sim 5-6.5 \\
\sim 0.1-1.2 \\
\sim 1.5-5.0 \\
\sim 2-3 \\
0.0-4.0 \\
2.7-3.2 \\
0.0-0.1 \\
0.2-4.0 \\
0.015-3.0\end{array}$ & $\begin{array}{c}10^{43}<L_{2-8}<10^{45} \mathrm{erg} \mathrm{s}^{-1} \\
10^{42}<L_{2-8}<10^{46} \mathrm{erg} \mathrm{s}^{-1} \\
10^{42}<L_{2-8}<10^{46} \mathrm{erg} \mathrm{s}^{-1} \\
10^{43}<L_{2-8}<10^{44.5} \mathrm{erg} \mathrm{s}^{-1} \\
10^{42}<L_{2-10}<10^{46.5} \mathrm{erg} \mathrm{s}^{-1} \\
10^{43}<L_{2-10}<10^{44.5} \mathrm{erg} \mathrm{s}^{-1} \\
10^{41}<L_{3-20}<10^{46} \mathrm{erg} \mathrm{s}^{-1} \\
10^{42}<L_{0.3-8}<10^{45.5} \mathrm{erg} \mathrm{s}^{-1} \\
10^{41.5}<L_{2-10}<10^{46.5} \mathrm{erg} \mathrm{s}^{-1}\end{array}$ & $\begin{array}{c}1.02 / 1 \\
41.0 / 30 \\
15.5 / 9 \\
1.73 / 1 \\
14.4 / 18 \\
0.77 / 1 \\
9.75 / 10 \\
26.3 / 15 \\
26.5 / 35\end{array}$ & $\begin{array}{c}1 \\
601 \\
\sim 100 \\
136 \\
508 \\
15 \\
77 \\
368 \\
247\end{array}$ & $\begin{array}{c}\text { diamonds } \\
\text { squares } \\
\ldots \\
\ldots \\
\text { stars } \\
\text { crosses } \\
\text { inverted triangles } \\
\text { triangles } \\
\text { circles }\end{array}$ \\
\hline \multicolumn{8}{|l|}{ Mid-IR: } \\
\hline $\begin{array}{l}\text { Brown et al. (2006) } \\
\text { Matute et al. (2006) } \\
\text { Emission Lines: }\end{array}$ & $\begin{array}{l}\text { Spitzer Boötes (NDWFS) } \\
\text { RMS + ELIAS + HDF-N/S }\end{array}$ & $\begin{array}{c}8 \mu \mathrm{m} \\
15 \mu \mathrm{m}\end{array}$ & $\begin{aligned} & \sim 1-5 \\
& \sim 0.1-1.2\end{aligned}$ & $\begin{array}{c}10^{45}<L_{8 \mu \mathrm{m}}<10^{47} \mathrm{erg} \mathrm{s}^{-1} \\
10^{42}<L_{15 \mu \mathrm{m}}<10^{47} \mathrm{erg} \mathrm{s}^{-1}\end{array}$ & $\begin{array}{l}3.77 / 10 \\
23.4 / 18\end{array}$ & $\begin{array}{l}183 \\
148\end{array}$ & $\begin{array}{l}\text { circles } \\
\text { squares }\end{array}$ \\
\hline $\begin{array}{l}\text { Hao et al. (2005) } \\
\ldots \\
\ldots\end{array}$ & $\begin{array}{l}\text { SDSS (main galaxy sample) } \\
\ldots \\
\ldots\end{array}$ & $\begin{array}{l}\mathrm{H} \alpha \\
{[\mathrm{OII}]} \\
{[\mathrm{OIII}]}\end{array}$ & $\begin{array}{c}0-0.33 \\
\ldots \\
\ldots\end{array}$ & $\begin{aligned} 10^{5}<L_{\mathrm{H} \alpha} & <10^{9} L_{\odot} \\
10^{5}<L_{\mathrm{OII}} & <10^{8} L_{\odot} \\
10^{5} & <L_{\mathrm{OIII}}<10^{9} L_{\odot}\end{aligned}$ & $\begin{array}{l}29.5 / 21 \\
\quad \ldots \\
\quad \cdots\end{array}$ & $\begin{array}{c}\sim 3000 \\
\quad \ldots \\
\quad \ldots\end{array}$ & $\begin{array}{c}\text { pentagons } \\
\ldots \\
\ldots\end{array}$ \\
\hline
\end{tabular}

${ }^{a}$ For a detailed description of each sample, we direct the reader to the listed references (and references therein).

${ }^{b}$ Redshift and luminosity ranges listed are for the entire sample in each case, they should not be taken to imply that the observations simultaneously span both ranges.

${ }^{\mathrm{c}}$ Reduced $\chi^{2}$ of binned QLF with respect to our full best-fit. 
TABLE 2

Best-Fit QLF AT VARIOUs REDSHIFTS

\begin{tabular}{cccccc}
\hline \hline$\langle z\rangle$ & $\log \phi_{*}{ }^{\mathrm{a}}$ & $\log L_{*}{ }^{\mathrm{b}}$ & $\gamma_{1}$ & $\gamma_{2}$ & $\chi^{2} / \nu$ \\
\hline 0.1 & $-5.45 \pm 0.28$ & $11.94 \pm 0.21$ & $0.868 \pm 0.050$ & $1.97 \pm 0.17$ & $89 / 73$ \\
0.5 & $-4.66 \pm 0.26$ & $12.24 \pm 0.18$ & $0.600 \pm 0.136$ & $2.26 \pm 0.23$ & $124 / 66$ \\
1.0 & $-4.63 \pm 0.15$ & $12.59 \pm 0.11$ & $0.412 \pm 0.122$ & $2.23 \pm 0.15$ & $182 / 69$ \\
1.5 & $-4.75 \pm 0.19$ & $12.89 \pm 0.13$ & $0.443 \pm 0.145$ & $2.29 \pm 0.20$ & $214 / 86$ \\
2.0 & $-4.83 \pm 0.05$ & $13.10 \pm 0.04$ & $0.320 \pm 0.046$ & $2.39 \pm 0.07$ & $66 / 67$ \\
2.5 & $-4.96 \pm 0.14$ & $13.13 \pm 0.09$ & $0.302 \pm 0.091$ & $2.30 \pm 0.15$ & $72 / 53$ \\
3.0 & $-5.23 \pm 0.12$ & $13.17 \pm 0.10$ & $0.395 \pm 0.060$ & $2.10 \pm 0.12$ & $45 / 53$ \\
4.0 & $-4.66 \pm 0.37$ & $12.39 \pm 0.32$ & $-0.254 \pm 0.736$ & $1.69 \pm 0.18$ & $54 / 32$ \\
5.0 & $-5.38 \pm 1.19$ & $12.46 \pm 1.10$ & $0.497 \pm 0.458$ & $1.57 \pm 0.41$ & $14 / 13$ \\
6.0 & $-5.13 \pm 0.38$ & 11.0 & 0.0 & $1.11 \pm 0.13$ & $5 / 3$ \\
& & & & & \\
\hline
\end{tabular}

${ }^{\mathrm{a}} \mathrm{Mpc}^{-3}$

${ }^{\mathrm{b}} L_{\odot} \equiv 3.9 \times 10^{33} \mathrm{erg} \mathrm{s}^{-1}$

TABLE 3

BEST-Fit QLF TO ALL REDSHIFTS

\begin{tabular}{|c|c|c|c|c|c|c|c|c|c|c|c|c|}
\hline Model & $\log \phi_{*}{ }^{\mathrm{a}}$ & $\left(\log L_{*}\right)_{0}{ }^{\mathrm{b}}$ & $k_{L, 1}$ & $k_{L, 2}$ & $k_{L, 3}$ & $\left(\gamma_{1}\right)_{0}$ & $k_{\gamma_{1}}$ & $\left(\gamma_{2}\right)_{0}$ & $k_{\gamma_{2}, 1}$ & $k_{\gamma_{2}, 2}$ & $\rho_{\mathrm{BH}}(z=0)^{\mathrm{c}}$ & $\chi^{2} / \nu$ \\
\hline PLE & $-4.733 \pm 0.101$ & $12.965 \pm 0.074$ & $0.749 \pm 0.084$ & $-8.03 \pm 0.35$ & $-4.40 \pm 1.05$ & $0.517 \pm 0.065$ & 0 & $2.096 \pm 0.083$ & 0 & 0 & 5.66 & $1924 / 511$ \\
\hline Faint & $-4.630 \pm 0.075$ & $12.892 \pm 0.058$ & $0.717 \pm 0.069$ & $-8.10 \pm 0.29$ & $-3.90 \pm 0.85$ & $0.272 \pm 0.073$ & $-0.972 \pm 0.268$ & $2.048 \pm 0.063$ & 0 & 0 & 4.97 & $1422 / 510$ \\
\hline Bright & $-4.930 \pm 0.070$ & $13.131 \pm 0.051$ & $0.360 \pm 0.095$ & $-11.63 \pm 0.48$ & $-10.68 \pm 1.04$ & $0.605 \pm 0.044$ & 0 & $2.350 \pm 0.087$ & $1.53 \pm 0.14$ & $-0.745 \pm 0.081$ & 5.47 & $1312 / 509$ \\
\hline Scatter $^{d}$ & $-4.815 \pm 0.040$ & $13.064 \pm 0.030$ & $0.356 \pm 0.061$ & $-11.69 \pm 0.27$ & $-9.18 \pm 0.58$ & $0.351 \pm 0.032$ & $-0.826 \pm 0.092$ & $2.359 \pm 0.052$ & $1.534 \pm 0.070$ & $-0.889 \pm 0.042$ & 4.63 & $445 / 508$ \\
\hline Schechter ${ }^{\mathrm{e}}$ & $-3.579 \pm 0.092$ & $11.482 \pm 0.162$ & $-1.78 \pm 0.29$ & $-21.22 \pm 1.62$ & $-25.93 \pm 3.08$ & $0.013 \pm 0.011$ & $-3.760 \pm 0.859$ & $0.354 \pm 0.020$ & $1.794 \pm 0.087$ & $-0.784 \pm 0.063$ & 1.16 & $1254 / 507$ \\
\hline Full & $-4.825 \pm 0.060$ & $13.036 \pm 0.043$ & $0.632 \pm 0.077$ & $-11.76 \pm 0.38$ & $-14.25 \pm 0.80$ & $0.417 \pm 0.055$ & $-0.623 \pm 0.132$ & $2.174 \pm 0.055$ & $1.460 \pm 0.096$ & $-0.793 \pm 0.057$ & 4.81 & $1007 / 508$ \\
\hline
\end{tabular}

${ }^{\mathrm{a}} \mathrm{Mpc}^{-3}$

${ }^{\mathrm{b}} L \odot 3.9 \times 10^{33} \mathrm{erg} \mathrm{s}^{-1}$

${ }^{\mathrm{c}} 10^{5} \mathrm{M} \mathrm{M}_{\odot} \mathrm{Mpc}^{-3}$

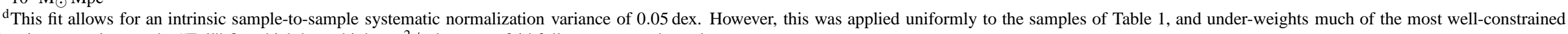
data in comparison to the "Full" fit, which has a higher $\chi^{2} / \nu$ but more faithfully represents those data sets.

Adopting the modified Schechter function fit of Equation 21 instead of a double power law.

The resulting observed QLF at arbitrary frequency and redshift from any of these fits can be calculated using the QLF calculator script available for download at http://www.cfa.harvard.edu/ phopkins/Site/qlf.html 
TABLE 4

BEST-FIT LDDE QLF

\begin{tabular}{|c|c|c|c|c|c|c|c|c|c|c|c|c|c|}
\hline Model & $\log \phi_{*}{ }^{\mathrm{a}}$ & $\log L_{*}{ }^{\mathrm{b}}$ & $\gamma_{1}$ & $\gamma_{2}$ & $\log L_{c}{ }^{\mathrm{b}}$ & $z_{c 0}$ & $\alpha$ & $p 1_{46}$ & $p 2_{46}$ & $\beta_{1}$ & $\beta_{2}$ & $\rho_{\mathrm{BH}}(z=0)^{\mathrm{c}}$ & $\chi^{2} / \nu$ \\
\hline PDE & $-6.66 \pm 0.28$ & $46.64 \pm 0.19$ & $0.858 \pm 0.075$ & $2.09 \pm 0.20$ & 46 & $1.852 \pm 0.068$ & 0 & $4.13 \pm 0.29$ & $-2.53 \pm 0.46$ & 0 & 0 & 9.78 & $3255 / 511$ \\
\hline LDDE & $-6.20 \pm 0.15$ & $45.99 \pm 0.10$ & $0.933 \pm 0.045$ & $2.20 \pm 0.14$ & $46.72 \pm 0.05$ & $1.852 \pm 0.025$ & $0.274 \pm 0.025$ & $5.95 \pm 0.23$ & $-1.65 \pm 0.21$ & $0.29 \pm 0.34$ & $-0.62 \pm 0.17$ & 5.09 & $1389 / 507$ \\
\hline
\end{tabular}

${ }^{\mathrm{a}} \mathrm{Mpc}^{-3}$

${ }^{\mathrm{a}} \mathrm{Mpc}^{-3}$

${ }^{c} 10^{5} \mathrm{M}_{\odot} \mathrm{Mpc}^{-3}$. In this formulation, the luminosity density is nearly divergent at low $L$, so the integration is truncated at $10^{41} \mathrm{erg} \mathrm{s}^{-1}$.

The resulting observed QLF at arbitrary frequency and redshift from any of these fits can be calculated using the QLF calculator script available for download at

http://www.cfa.harvard.edu/ phopkins/Site/qlf.html

TABLE 5

Best-Fit Quasar Formation Rate

\begin{tabular}{|c|c|c|c|c|c|c|c|c|c|c|c|}
\hline Model & $\left(\log \dot{\phi}_{*}\right)_{0}^{\mathrm{a}}$ & $k_{\dot{\phi}}$ & $\left(\log M_{*}\right)_{0}^{\mathrm{b}}$ & $k_{M, 1}$ & $k_{M, 2}$ & $\overline{\eta_{1}}{ }^{\mathrm{c}}$ & $\left(\eta_{2}\right)_{0}$ & $k_{\eta_{2}, 1}$ & $k_{\eta_{2}, 2}$ & $\rho_{\mathrm{BH}}(z=0)^{\mathrm{d}}$ & $\overline{\chi^{2} / \nu}$ \\
\hline Fixed $\gamma_{2}$ & $-3.808 \pm 0.037$ & $-4.46 \pm 0.31$ & $8.894 \pm 0.037$ & $1.54 \pm 0.12$ & $-4.46 \pm 0.31$ & 0.20 & $2.44 \pm 0.11$ & 0 & 0 & 6.19 & $1338 / 511$ \\
\hline Variable $\gamma_{2}$ & $-3.830 \pm 0.031$ & $-4.02 \pm 0.36$ & $8.959 \pm 0.032$ & $1.18 \pm 0.13$ & $-6.68 \pm 0.44$ & 0.20 & $2.86 \pm 0.16$ & $1.80 \pm 0.18$ & $-1.13 \pm 0.09$ & 6.28 & $1206 / 509$ \\
\hline Schechter ${ }^{\mathrm{e}}$ & $-3.571 \pm 0.080$ & $-3.81 \pm 0.46$ & $8.710 \pm 0.130$ & $1.33 \pm 0.21$ & $-7.64 \pm 0.99$ & 0.20 & $0.79 \pm 0.10$ & $1.33 \pm 0.19$ & $-1.17 \pm 0.12$ & 4.27 & $1434 / 509$ \\
\hline
\end{tabular}

${ }^{\mathrm{a}} \mathrm{Mpc}^{-3} \mathrm{Gyr}^{-1}$

${ }^{\mathrm{c}}$ In this model, the QLF faint-end slope is dominated by the shape of the quasar lightcurve, giving only weak constraints on $\eta_{1}$. We adopt the maximum acceptable $\eta_{1} \approx 0.20$ $(1 \sigma)$ as an upper limit, and show the fit results for this case.

${ }^{\mathrm{d}} 10^{5} \mathrm{M} \odot \mathrm{Mpc}^{-3}$

${ }^{\mathrm{e}}$ Adopting the modified Schechter function fit of Equation 21 instead of a double power law. 